\title{
Stichproben für Telefonbefragungen in Deutschland: Ein Werkstattbericht
}

\author{
Christian Strippel \& Martin Emmer
}

\section{Einleitung}

Das Thema Stichprobenziehung für bevölkerungsrepräsentative Befragungen in Deutschland hat in der wissenschaftlichen Diskussion seit etwa zwei Jahren wieder Konjunktur. Allgemeiner Hintergrund ist die mittlerweile annähernde Vollversorgung der deutschen Bevölkerung mit Internetzugängen und die damit einhergehende Möglichkeit internetbasierter Befragungen. Den konkreten Anlass für die Diskussion lieferte schließlich das Meinungsforschungsinstitut Civey, das für die Ergebnisse seiner gänzlich auf selbstrekrutierten Internet-Panels basierenden Befragungen den Anspruch auf Repräsentativität erhebt und damit steigende Popularität insbesondere unter großen Medienhäusern genießt. ${ }^{1}$ Durch eine Kombination aus dem sogenannten „River Sampling“ (OLIVER 2011), bei dem die Befragten über eine Vielzahl von Internetseiten rekrutiert werden, und verschiedenen Gewichtungsverfahren in der Datenauswertung versucht das Unternehmen, aus den von ihnen erhobenen Umfragedaten bevölkerungsrepräsentative Statistiken zu errechnen (siehe CIVEY 2020, BOUNCKEN 2018). Kritisiert wird dieses Vorgehen bzw. die damit verbundene Repräsentativitätsbehauptung nicht nur von konkurrierenden Meinungsforschungsinstituten (ADM 2018, THIERHOFF 2018), sondern auch von Vertretern der empirischen Sozialforschung (etwa FRIES 2018, SCHNELL 2018). Der zentrale Kritikpunkt lautet: Ohne eine Zufallsauswahl der Befragten, durch die allein garantiert werden könne, dass alle Elemente der zu untersuchenden Population eine berechenbare Chance haben, in der Auswahl berücksichtigt zu werden, kann - anders als bei der bisher dominierenden Methode der Telefonbefragung - nicht von einer Repräsentativität ausgegangen werden.

Der Kritik an Civey gegenüber steht in dieser Diskussion der Zweifel, dass die theoretischen Anforderungen an die Zufallsauswahl aufgrund der in den vergangenen Jahren stetig sinkenden Ausschöpfungsquoten selbst in Telefonumfragen noch erreicht werden können (siehe

1 Nach Angaben von Civey haben bislang etwa Der Spiegel, Welt, Süddeutsche Zeitung, Tagesspiegel, Wirtschaftswoche, Focus und Phoenix mit dem Unternehmen zusammengearbeitet (www.civey.com/media). 
etwa RENDTEL 2018, WILDNER 2020). Insofern könne es sich, so argumentiert die Verteidigung alternativer Verfahren, bei dem Konstrukt der Bevölkerungsrepräsentativität in der Forschungspraxis sowieso nur (noch) um einen unerreichbaren Anspruch handeln, auf den lediglich mehr oder weniger erfolgreich hingearbeitet werden könne. So müsse gerade in der kommerziellen bzw. angewandten Markt- und Meinungsforschung abgewogen werden zwischen den qualitativen Anforderungen an die Stichprobenziehung und den hohen Kosten, die mit solchen Qualitätsanforderungen verbunden seien.

Vor dem Hintergrund dieser Diskussion werden in dem vorliegenden Beitrag die theoretischen Standards und Empfehlungen für Stichprobenziehungen bei Telefonbefragungen daraufhin untersucht, inwieweit sie heute noch eine geeignete Basis für bevölkerungsrepräsentative Befragungsstudien darstellen. Konkret werden dazu unter Rückgriff auf entsprechende Methodenliteratur im nächsten Abschnitt die Vorgaben und Empfehlungen für drei zentrale Aspekte der Stichprobenziehung bei Telefonbefragungen theoretisch vorgestellt. Eingegangen wird dabei erstens auf das Stichprobendesign bzw. die Zusammensetzung der Auswahlrahmen für Festnetz- und Mobilfunkstichproben; zweitens auf die Stichprobenbereinigung, wobei sich auf die technische Validierung von Mobilfunknummern mithilfe des sogenannten „Home Local Register Lookup“-Verfahrens (kurz: HLR-Lookup) konzentriert wird; und drittens auf die theoretischen Anforderungen an die Stichprobenausschöpfung. Anschließend wird die forschungspraktische Umsetzung dieser Empfehlungen anhand einer im Herbst 2019 vom Weizenbaum-Institut für die vernetzte Gesellschaft in Kooperation mit dem Institut für Publizistik- und Kommunikationswissenschaft der Freien Universität Berlin durchgeführten Telefonbefragung zu Bürgernormen und politischer Partizipation in Deutschland evaluiert. Im letzten Abschnitt werden daraus Schlüsse für die Forschungspraxis zukünftiger Studien gezogen.

\section{Standards und Empfehlungen}

Die Methodenliteratur zum Thema Stichprobenziehung für Telefonbefragungen ist aufgrund der seit Jahrzehnten zentralen Stellung der Methode in der kommerziellen und wissenschaftlichen Sozialforschung so umfangreich, dass eine vollständige Aufarbeitung der im Laufe der Zeit entwickelten Standards und Empfehlungen an dieser Stelle nicht zu leisten ist. Aus diesem Grund stützt sich die folgende Darstellung auf jene Überblicksliteratur, die solche Zusammenfassungen bereits vorgenommen hat. Da zwar die theoretischen Anforderungen an Stichproben universell sind, für die praktische Ziehung aber der konkrete Kontext - etwa das Telefonnummernsystem - relevant ist, wird dabei vor allem jene Literatur berücksichtigt, die sich der Ziehung von Telefonstichproben in Deutschland widmet. In diesem Bereich haben insbesondere das 1974 gegründete "Zentrum für Umfragen, Methoden und Analysen“ (ZUMA) sowie ab 2008 das „GESIS - Leibniz-Institut für Sozialwissenschaften“ (als Folgeinstitution) wegweisende Arbeit geleistet. Mit den hier angesiedelten Abteilungen „Dauerbeobachtung der Gesellschaft" und "Survey Design and Methodology" stellt die GESIS die wichtigste Forschungsinfrastruktur für sozialwissenschaftliche Umfrageforschung in Deutschland dar. Der jüngste Methodenüberblick zum Thema "Telefonumfragen in Deutschland“ aus diesem Haus (HÄDER/ HÄDER/SCHMICH 2019) ist noch recht aktuell und dient im Folgenden deshalb als Ausgangspunkt und zentrale Referenz für die Aufarbeitung der Standards und Empfehlungen in den drei bereits genannten Bereichen Stichprobenziehung, -bereinigung und -ausschöpfung. Maßgeblich ist dabei der Beitrag zu „Telefonstichproben“ von HÄDER und SAND (2019). 


\subsection{Stichprobenziehung}

Die Frage danach, wie Rufnummern für bevölkerungsrepräsentative Telefonbefragungen gezogen werden müssen, um zu gewährleisten, dass alle Personen eine berechenbare Wahrscheinlichkeit haben, angerufen und befragt zu werden, zählt zu den wichtigsten und entsprechend intensiv erforschten Problemen in der Umfrageforschung. Die Literatur zu dieser Frage reicht weit zurück bis zu den Anfängen dieses Forschungszweigs (BOWLEY 1936, GALLUP 1944, eine ausführliche Darstellung findet sich bei KELLER 2001). Für Deutschland datiert ein Großteil der in dieser Frage heute noch maßgeblichen Forschung auf die 1990er und frühen 2000er Jahre. In dieser Zeit haben sich zwei für die Stichprobenziehung entscheidende Veränderungen ergeben: Zum einen sank die Eintragsdichte des Telefonbuches nach Aufhebung der Eintragungspflicht 1991 erheblich, sodass es als zuverlässige Auswahlgrundlage für die Stichprobenziehung für Festnetzrufnummern ausschied (HÄDER 1994, 1996, 2000, MARHENKE 1997, FOLLMER/SMID 1998, DEUTSCHMANN/HÄDER 2002). Entscheidend ist dabei der Umstand, so HÄDER und SAND (2019), dass „sich Personen aus eingetragenen und nichteingetragenen Haushalten mit Festnetztelefon hinsichtlich soziodemographischer Variablen" (S. 47) wie Alter und Erwerbsstatus unterscheiden, wodurch es „bei einer Ziehung aus dem Telefonbuch zu systematisch verzerrten Bruttostichproben“ (ebd.) komme.

Abbildung 1: Anzahl der registrierten Mobilfunknummern in Deutschland, 1992-2019

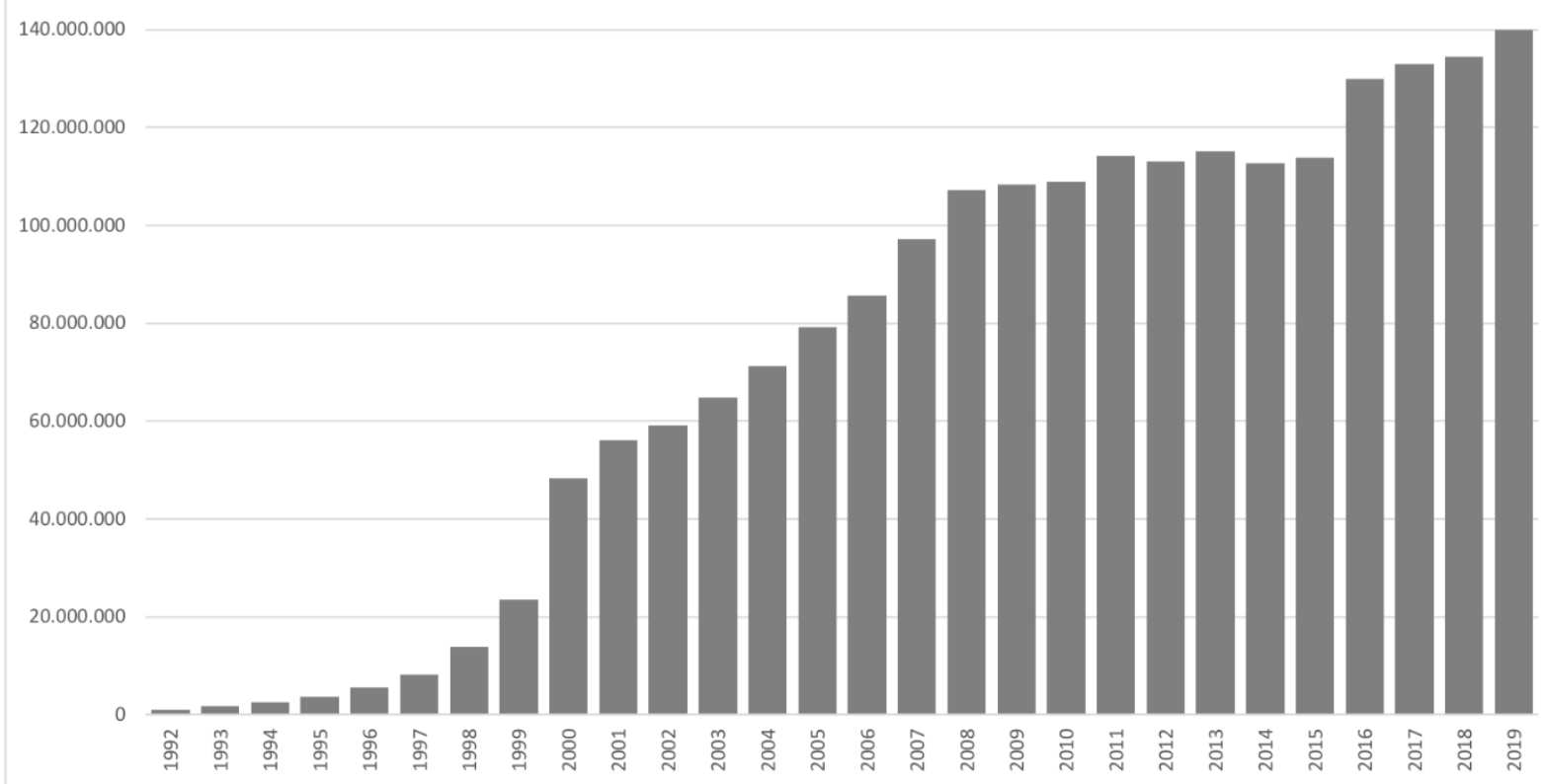

Anmerkung: Es werden hier jeweils die Daten für das 4. Quartal eines Jahres angegeben. Der sprunghafte Anstieg der Zahlen in 2016 ist auf Veränderungen in der Zäh/weise bei Vodafone zurückzuführen. Datenquelle: BUNDESNETZAGENTUR (2020).

Zum anderen nahm die Mobilfunknutzung um die Jahrtausendwende sprunghaft zu (siehe Abbildung 1), wodurch die mobile Erreichbarkeit eines wachsenden Teils der Bevölkerung in Deutschland im Zuge der Stichprobenziehung berücksichtigt werden musste (GRUND 2002, GLEMSER 2007, SCHNEIDERAT/SCHLINZIG 2009, BUSSE/FUCHS 2015). Insbesondere galt es, ein Undercoverage der sogenannten „Mobile-onlys" zu vermeiden, also jener Bevölkerungsgruppen, die nur (noch) auf dem Mobiltelefon zu erreichen ist (BUSSE/FUCHS 2012, SCHNEIDERAT/ 
SCHLINZIG 2012). Denn auch hier gilt, dass „sich Festnetzhaushalte und Mobilfunkhaushalte hinsichtlich für die Sozialforschung relevanter Merkmale unterscheiden" (HÄDER/SAND 2019: $60)$, sodass sich bei Nichtberücksichtigung systematische Verzerrungen ergeben. So sind „Mobile-onlys" in Deutschland "häufiger jünger, eher männlich, stammen eher aus Ostdeutschland und leben eher als Singles" (ebd.: 61).

Zur Adressierung dieser Herausforderungen wurden neue Verfahren der Stichprobenziehung entwickelt, die auf die Besonderheiten der Rufnummernstruktur und der Telefonnutzung in Deutschland angepasst sind. Zunächst war es wichtig, so HÄDER und SAND (2019), „einen für Deutschland praktikablen, relativ leicht zu konstruierenden, unaufwändig zu aktualisierenden und von der Datenbasis her legal zugänglichen Auswahlrahmen für Festnetztelefonstichproben zu entwerfen" (S. 47), der das Telefonbuch als Grundlage für die Stichprobenziehung ablösen konnte. Bei einem Auswahlrahmen handelt es sich um eine Liste, „auf der die Elemente der Grundgesamtheit akzeptabel verzeichnet sind" (GABLER/HÄDER 2015: 3), bei der also Auswahl- und Grundgesamtheit möglichst übereinstimmen und die keine systematischen Abweichungen aufweist. Da das deutsche Rufnummernsystem sehr heterogen strukturiert ist, konnten die für die USA entwickelten Verfahren des „Random Digit Dialing“ (RDD) und „Randomized Last Digits" (RLD), bei denen ausgewählte Ziffern von im Telefonbuch gelisteten Nummer zufällig generiert und ausgetauscht werden, nicht ohne Weiteres auf Deutschland angewandt werden (FREY/KUNZ/LÜSCHEN 1990: 94 ff., GABLER/HÄDER 1998, HÄDER/SAND 2019: 50 f.). Aus diesem Grund wurde Mitte der 1990er Jahre am ZUMA in Mannheim ein mehrstufiges Stichprobendesign entwickelt, das sich seither unter der Bezeichnung "GablerHäder-Design" (nach GABLER/HÄDER 1997, 1999, HÄDER/GABLER 1998) im akademischen Bereich als Auswahlrahmen für Festnetzstichproben in Deutschland durchgesetzt hat (HÄDER 2000, GABLER/SCHÜRLE 2002, HÄDER 2015, HÄDER/SAND 2019: 51 ff.). Für die kommerzielle Forschung hat der Arbeitskreis Deutscher Markt- und Sozialforschungsinstitute (ADM) auf der Grundlage dieses Stichprobendesigns einen ähnlichen Auswahlrahmen entwickelt (HECKEL 2002, 2007, VON DER HEYDE 2002, GLEMSER 2002, ADM 2014).

Die Konstruktion des Auswahlrahmens nach dem Gabler-Häder-Design folgt, grob gesagt, drei Schritten (siehe dazu HÄDER/SAND 2019: 51 ff.): Zuerst werden unter Rückgriff auf das Telefonbuch alle dort gelisteten Rufnummern erhoben und jene Nummern gelöscht, „die offensichtlich nicht zur Grundgesamtheit der Wohnbevölkerung" (S. 51) gehören. Dazu zählen etwa alle als Geschäftsanschlüsse erkennbare Rufnummern, die bei allgemeinen Bevölkerungsumfragen in der Regel nicht berücksichtigt werden. Im zweiten Schritt werden die verbliebenen Rufnummern nach den 5.200 Ortsnetzbereichen in Deutschland sortiert und dort jeweils in 100er Blöcke eingeteilt. Zuletzt werden alle Blöcke innerhalb eines Ortsnetzbereichs, für die im Telefonbuch nicht mindestens eine Rufnummer gelistet ist, aussortiert und für die anderen Blöcke „alle 100 möglichen Ziffernfolgen generiert" (S. 52). Zusammen mit den bereits im Telefonbuch eingetragenen Nummern ergeben die so generierten Nummern jenen Auswahlrahmen, aus dem dann zufällig eine Stichprobe gezogen werden kann. Da durch dieses Vorgehen auch zahlreiche Rufnummern in den Auswahlraumen aufgenommen werden, die gar nicht geschaltet sind, ist die auf diese Weise konstruierte Auswahlgesamtheit um ein Vielfaches größer als die Grundgesamtheit aller geschalteten Festnetznummern. Der von der GESIS nach dem Gabler-Häder-Design erstellte Auswahlrahmen umfasste im Juli 2016 zum Beispiel knapp 178,8 Millionen Festnetznummern, von den 16,7 Millionen Nummern im Telefonbuch eingetragen sind und weitere 162,2 Millionen Nummern nach dem oben beschriebenen Verfahren generiert wurden (siehe Abbildung 2). 
Abbildung 2: Universum an Festnetznummern in Deutschland inklusive Auswahlrahmen nach dem Gabler-Häder-Design (Stand: Juli 2016)

Aussortierte Nummernblöcke
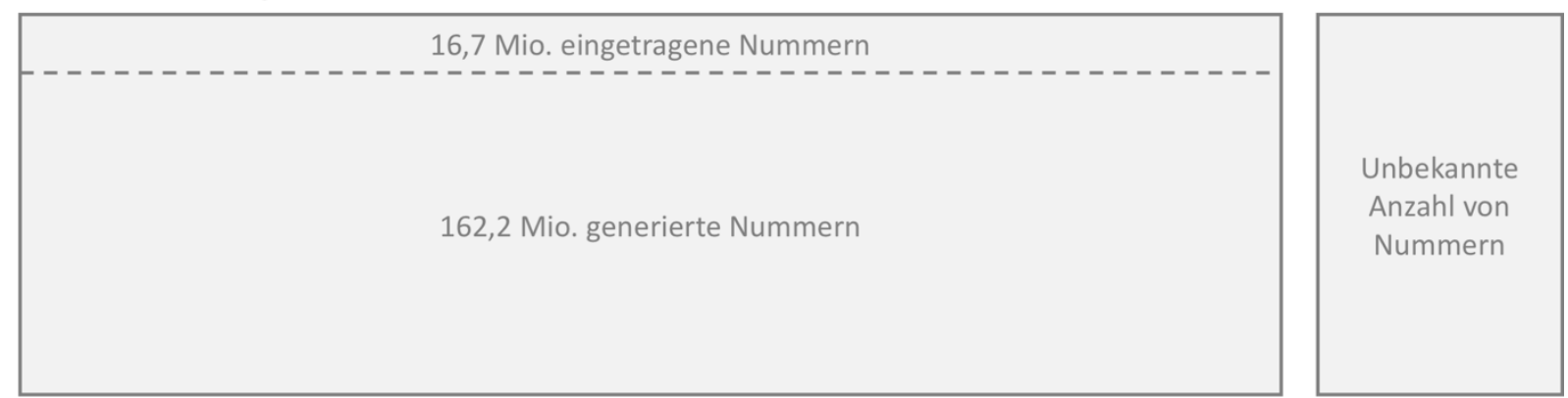

Anmerkung: Die Auswahlgesamtheit umfasst knapp 178,8 Mio. Festnetznummern, von denen 9,3\% aus dem Telefonbuch entnommen und 90,7\% generiert wurden. Datenquelle: HÄDER/SAND (2019: 54).

Die zweite grundlegende Veränderung im Bereich der Stichprobenziehung für Telefonumfragen in Deutschland bestand im Miteinbeziehen von Mobilfunknummern, um auch die allmählich wachsende Gruppe der "Mobile-onlys" zu erreichen (siehe GABLER/HÄDER 2005). Da nur ein kleiner Bruchteil der Mobilfunknummern im Telefonbuch eingetragen ist und auch keine sonstigen Listen zur Verfügung stehen, auf die für eine Stichprobenziehung zurückgegriffen werden könnte (siehe HÄDER/GLEMSER 2006: $163 \mathrm{ff}$.), wird der Auswahlrahmen im RDDVerfahren „so konstruiert, dass für alle gültigen Vorwahlbereiche (also z.B. 0171, 0165, 0177) alle sinnvollen Nummernfolgen generiert werden" (HÄDER/SAND 2019: 62). Mit aktuell rund 212,2 Millionen Rufnummern (siehe ebd.) ist dieser Auswahlrahmen dadurch noch einmal erheblich größer als der Auswahlrahmen für Festnetznummern. Ein besonderes Problem beim Abtelefonieren von Mobilfunkstichproben ergibt sich dabei vor allem dadurch, dass der Anteil von erreichten Anrufbeantwortern in der Regel vergleichsweise hoch ausfällt, dieses Anrufergebnis jedoch „,kein hinreichend verlässlicher Indikator dafür ist, dass hinter dem digitalen Anrufbeantworter auch ein aktiv geschalteter Anschluss steht" (HÄDER/SAND 2019: 64). Für Unsicherheit sorgt hier in erster Linie der Umstand, dass viele Mobilfunkanschlüsse, obwohl sie noch gar nicht oder nicht mehr genutzt werden, dennoch geschaltet sind, weshalb entsprechende Anrufe dann zu der Standard-Mailbox des jeweiligen Providers führen (siehe HÄDER ET AL. 2009: S. 73). Dadurch haben viele der generierten Mobilfunknummern auch nach mehreren Kontaktversuchen, die immer wieder zu einem Anrufbeantworter führen, einen unklaren Status. Wie nach der maximalen Anzahl von zehn Erstkontaktversuchen (siehe ADM 2016) und im Zuge der Berechnung der Stichprobenausschöpfung bzw. Responserate mit solchen Fällen umzugehen ist, darüber herrscht laut HÄDER und SAND (2019) „unter den Umfrageforschern noch keine Einigkeit" (S. 66). Studien, die um eine Klärung dieser Frage bemüht sind, liegen noch keine vor.

Eine reine Mobilfunkstichprobe würde in Deutschland derzeit ebenfalls noch zu systematischen Verzerrungen führen, da nach wie vor ein signifikanter Anteil der Bevölkerung keinen Mobilfunkanschluss hat und deshalb nur über das Festnetz zu erreichen ist (SCHNEIDERAT/ SCHLINZIG 2012). Bei diesen "Landline-onlys" handelt es sich laut HÄDER und SAND (2019) „häufiger um ältere, auf dem Land in Westdeutschland lebende Personen“ (S. 62). Um eine bevölkerungsrepräsentative Stichprobe für Telefonumfragen in Deutschland zu ziehen, müssen deshalb die beiden Auswahlrahmen für Festnetz- und Mobilfunknummern miteinander zu einem "Dual-Frame-Ansatz" kombiniert werden (HOFFMANN 2007, GABLER/AYHAN 2007, HUNSICKER/SCHROTH 2007, 2014, GABLER/HÄDER 2009, CALLEGARO ET AL. 2011, SAND 2014, 
GLEMSER/MEIER/HECKEL 2014). ${ }^{2}$ Die Festnetz- und Mobilfunkstichproben werden dabei unabhängig voneinander jeweils aus den beiden Auswahlrahmen gezogen. Auf der Grundlage einer vom ADM (2012) durchgeführten Simulation wird in diesem Zusammenhang ein Mischverhältnis von 70 Prozent Festnetz- und 30 Prozent Mobilfunknummern empfohlen (siehe etwa HÄDER/SAND 2019: 71). In dem entsprechenden Forschungsbericht zu der Simulationsstudie heißt es dazu: „Diese Verteilung sollte angestrebt werden. Zwar wäre ein Verhältnis von 60 Prozent Festnetznummern zu 40 Prozent Mobilfunknummern optimal, die nur geringfügigen Gewinne bei der Effizienz rechtfertigen aus unserer Sicht die höheren Kosten allerdings eher nicht" (ADM 2012: 31).

\subsection{Stichprobenbereinigung}

Im Zuge der Konstruktion der beiden Auswahlrahmen für Festnetz- und Mobilfunknummern wird in beiden Fällen eine große Zahl nicht vergebener Rufnummern erzeugt. Wie dargestellt, fallen dabei insbesondere Mobilfunknummern ins Gewicht: „Durch die Unterschiede in der Konstruktion der jeweiligen Auswahlrahmen“, so HÄDER und SAND (2019), „ist die Anzahl der ungeschalteten Rufnummern für Stichproben von Mobilfunknummern per Definition höher als für Stichproben von Festnetznummern" (S. 66). Für Deutschland wird ihr Anteil auf etwa 40 Prozent geschätzt (STRUMINSKAYA ET AL. 2011: 2). Hinzu kommen - aufgrund der angesprochenen Probleme mit Anrufbeantwortern - „die deutlich höheren Schwierigkeiten (sowohl für automatische Dialer als auch für Interviewer) eindeutig festzustellen, ob eine Mobilfunkrufnummer geschaltet ist oder nicht" (HÄDER/SAND 2019: 66). Diese Eigenheiten der Mobilfunkstichprobe führen nicht nur zu Problemen bei der Errechnung der Stichprobenausschöpfung bzw. Responserate, sondern auch zu erhöhten Telefoniekosten und einem belastenden Mehraufwand für die Interviewer*innen, die mitunter von einem Anrufbeantworter zum nächsten geleitet werden (siehe dazu GUTERBOCK ET AL. 2011, AAPOR 2010: 95 ff.).

Um diesen Problemen zu begegnen, empfehlen HÄDER und SAND (2019), für alle gezogenen Mobilfunknummern ein so genanntes „HLR-Lookup“ durchzuführen (S. 66-69), um die gezogene Mobilfunkbruttostichprobe im Vorfeld der Feldphase zu bereinigen (ähnlich auch ADM 2014: 175 f.). Dabei handelt es sich um ein Verfahren, mit dem die Mobilfunkstichprobe über eine technische Schnittstelle mit dem „Home Location Register" (HLR), einer Datenbank mit den Stammdaten aller Kunden der Mobilfunkanbieter in Deutschland, automatisiert abgeglichen wird (siehe STRUMINSKAYA ET AL. 2011, KUNZ/FUCHS 2012, SAND 2014, 2017). Auf diese Weise lässt sich ermitteln, ob die jeweiligen Mobilfunkrufnummern tatsächlich vergeben sind bzw. zu einem geschalteten Anschluss führen oder nicht; woraufhin die Mobilfunkstichprobe dann um alle nicht vergebenen Rufnummern bereinigt werden kann. Die zu prüfenden Mobilfunknummern werden dabei nicht gesondert angerufen, sodass kein Einfluss auf die eigentliche Befragung zu befürchten ist. Die vergleichsweise geringen Kosten, die für ein solches HLRLookup anfallen, werden schließlich durch erhöhte Kontakt- und Interviewraten - und damit auch eine verringerte Arbeitslast für die Interviewer*innen - um ein Vielfaches kompensiert (siehe STRUMINSKAYA ET AL. 2011: 4, KUNZ/FUCHS 2012: 380 ff.; SAND 2014: 17). Zudem konn-

2 Davon unabhängig besteht das Problem, dass ein großer Teil der Zielpersonen einer Befragung unter mehreren Rufnummern erreicht werden kann und für diese Personen damit eine höhere Wahrscheinlichkeit besteht, in der Stichprobe berücksichtigt zu werden. Diesem stichprobentheoretischen Problem wird in der Regel erst im Anschluss an die Erhebung durch eine entsprechende Gewichtung begegnet. In der Befragung selbst muss dazu die Anzahl der Festnetz- und Mobilfunknummern, unter denen die Befragten jeweils erreichbar sind, abgefragt werden (siehe SAND/GABLER 2019). 
ten nach einer solchen Bereinigung die Responseraten genauer bestimmt werden (STRUMINSKAYA ET AL. 2011: 4; KUNZ/FUCHS 2012: 384 ff.).

In einer 2015 durchgeführten Evaluationsstudie hat SAND (2017) die Reliabilität, Validität und Volatilität der HLR-Lookup-Verfahren verschiedener Anbieter untersucht. Zur Prüfung wurden insgesamt knapp 30.000 Mobilfunknummern - zu etwa gleichen Teilen von T-Mobile, Vodafone, Telefónica $\mathrm{O} 2$ und E-Plus - für ein Lookup an die beiden Anbieter MyCoolSMS und txtnation gegeben. Um die Volatilität der Abfrage zu testen und damit zu erfahren, „wie alt ein solches Lookup sein darf, um für eine Erhebung noch immer Gültigkeit zu besitzen" (S. 214), wurden beide Lookups nach zwei Wochen sowie nach weiteren vier Monaten noch einmal wiederholt. Die Validierung der Lookup-Ergebnisse erfolgte schließlich, indem eine Substichprobe von 12.129 Mobilfunknummern im Rahmen einer wissenschaftlichen Umfrage als Einsatzstichprobe verwendet wurde.

Der Abgleich der Lookup-Ergebnisse beider Anbieter zeigte, „dass neben der Kostenstruktur und der Art und Weise der berichteten Ergebnisse auch deutliche Unterschiede beim berichteten Status von überprüften Rufnummern bestehen" (S. 234). Aus diesem Grund empfiehlt SAND (2017), im Vorfeld einer Studie „auf die Wahl des Anbieters zu achten, da diese sich in den berichteten Ergebnissen sowie in der Qualität derselben deutlich unterscheiden können" (S. 235) und zudem „vor der Überprüfung der gesamten Brutto-Mobilfunkstichprobe durch einen bestimmten Anbieter eine Substichprobe zu testen, aus der evtl. Probleme ersichtlich werden können“ (ebd.). Der Vergleich über die drei Zeitpunkte hinweg zeigte „keine deutlichen Veränderungen zwischen der ersten und der zweiten Abfrage" (S. 225), allerdings hat in sieben Prozent der Fälle "ein Statuswechsel zwischen $t 1$ und $t 3$ stattgefunden" (S. 234). Vor diesem Hintergrund wird empfohlen, ein HLR-Lookup solle „möglichst zeitnah zum Feldbeginn durchgeführt werden und bei einer Feldzeit, die über mehrere Monate geht, für die (gesamte) Stichprobe wiederholt werden“ (S. 235). Damit soll vermieden werden, dass sich im Laufe einer Befragung der Status der Rufnummern ändert, etwa „wenn eine zuvor nicht geschaltete Rufnummer neu vergeben wird oder eine langfristig inaktive Rufnummer aus dem Netz ausgebucht wird“ (S. 215). Die Validierung des Lookups durch die Telefonumfrage zeigte schließlich, dass 0,5 (MyCoolSMS) bis 7,7 (txtnation) Prozent der Rufnummern ausgeschlossen wurden, die in der Umfrage jedoch zu einem geschalteten Anschluss führten. Um eine solche falsch-negative Selektion durch das Lookup zu vermeiden, wird empfohlen, „dass auch Rufnummern, deren Status als geschaltet und inaktiv gemeldet wird, in der Einsatzstichprobe enthalten bleiben" (S. 235).

\subsection{Stichprobenausschöpfung}

Die Qualität einer Stichprobe im Sinne ihrer Repräsentativität, also inwieweit sie als gute und damit strukturtreue Abbildung der angestrebten Grundgesamtheit gelten kann, bemisst sich schließlich zu einem großen Teil auch an der Ausschöpfungsquote (auch response rate oder Responserate). Sie gibt an, in welchem Umfang die Fälle, die sich in der zufällig gezogenen Stichprobe befinden, auch Eingang in den Datensatz finden - im Falle von Befragungen also, wie viele der ausgewählten und kontaktierten Personen am Ende befragt werden können (siehe etwa MÖHRING/SCHLÜTZ 2019: 34 f.). Eine perfekte Repräsentativität wäre im Prinzip nur dann gegeben, wenn sich tatsächlich jedes Element einer Grundgesamtheit anhand einer Stichprobe auch befragen lassen würde; sobald allerdings eine oder mehrere Personen ihre Teilnahme verweigern oder innerhalb der Feldzeit nicht erreicht werden können, ist ein Schluss von den Ergebnissen einer Stichprobenerhebung auf die Grundgesamtheit durch 
Unsicherheiten belastet (siehe dazu u.a. GABLER/HÄDER 2019). Ist dieser sogenannte „Nonresponse" (siehe SCHNELL 1997) zufällig verteilt, würde dies die Ergebnisse wenig beeinträchtigen; ist er allerdings hinsichtlich bestimmter Eigenschaften verzerrt - wenn also in stärkerem Maße Personen, die sich durch für die Befragung wichtige Eigenschaften auszeichnen, die Teilnahme verweigern -, sind auch die Ergebnisse der Befragung entsprechend verzert und damit die Schussfolgerungen gegebenenfalls fehlerhaft („Nonresponse Bias“, siehe KOCH/BLOHM 2015, SCHUPP/WOLF 2015).

Zur Berechnung der Stichprobenausschöpfung haben sich deshalb Standards durchgesetzt, die die errechneten Ausschöpfungsquoten auch vergleichbar machen. Als eine der wichtigsten Referenzen gelten hier die Standards der American Association for Opinion Research (AAPOR 2016), die in ähnlicher Form auch für Bevölkerungsbefragungen in Deutschland Anwendung finden (siehe dazu etwa GRAMLICH/LIEBAU/SCHUNTER 2019, STADTMÜLLER ET AL. 2019). In diesen Standards der AAPOR (2016) werden „response rates“ (RR) definiert als „the number of complete interviews with reporting units divided by the number of eligible reporting units in the sample" (S. 6). Zu ihrer Berechnung werden zunächst alle Fälle der abtelefonierten Stichprobe anhand ihrer jeweiligen Kontaktergebnisse („final disposition codes") in je eine der folgenden vier Kategorien eingeordnet: (1) „interviews", (2) „eligible cases that are not interviewed (non-respondents)“, (3) "cases of unknown eligibility“, und (4) "cases that are not eligible“ (alle Zitate S. 8). Welche Kontaktergebnisse dabei welcher dieser vier Gruppen zuzuordnen sind, ist detailliert festgelegt (S. 75, für den deutschen Kontext siehe STADTMÜLLER ET AL. 2019: 29 f.). Im zweiten Schritt lassen sich dann auf dieser Grundlage verschiedene Ausschöpfungsquoten berechnen (siehe GRAMLICHET AL. 2019: 306-313). Die zuverlässigsten sind die von der AAPOR (2016) definierte „minimum response rate“ (RRmin bzw. RR1) und „maximum response rate" (RRmax bzw. RR5), die zusammen die Spannweite der Stichprobenausschöpfung anzeigen. Bei RR1 wird die Anzahl der abgeschlossenen Interviews (1) durch die Summe der Kategorien (1), (2) und (3) geteilt, bei RR5 nur durch die Summe der Kategorien (1) und (2). Die entsprechenden Formeln lauten:

$$
\begin{gathered}
R R_{1}=\frac{(1)}{(1)+(2)+(3)}=R R_{\text {min }} \\
R R_{5}=\frac{(1)}{(1)+(2)}=R R_{\max }
\end{gathered}
$$

Die Berechnungen der beiden Responseraten unterscheiden sich also darin, wie jeweils mit den unbekannten Fällen, also den "cases of unknown eligibility“, umgegangen wird. Bei RR1 werden sie wie non-respondents (2) behandelt, bei RR5 wie die stichprobenneutralen Ausfälle aus Kategorie (4). Daneben definiert AAPOR (2016) noch drei weitere „response rates“: RR2 entspricht dabei RR1, nur dass neben den vollständigen Interviews auch die teilweise geführten Interviews mitgezählt werden. Analog gilt das auch für RR6 in Bezug auf RR5 (weshalb RR6 streng genommen das eigentliche RRmax ist). Bei RR3 handelt es sich um eine Responserate, bei der geschätzt wird, wie hoch der Anteil stichprobenneutraler Ausfälle in Kategorie (3) ausfällt. RR4 verhält sich dann zu RR3 analog zu RR2 und RR6.

Für die aktuelle Umfrageforschung besteht ein wesentliches Problem darin, dass die Bereitschaft von kontaktierten Personen zur Teilnahme an Befragungen seit Jahren abnimmt (STÖG BAUER 2000, CURTIN/PRESSER/SINGER 2005, GROVES 2006, AUST/SCHRÖDER 2009). An dem Beispiel der Panel-Befragungsstudie „Politische Online-Kommunikation“, die zwischen den 
Jahren 2000 und 2009 an der Technischen Universität Ilmenau in Kooperation mit der Heinrich-Heine-Universität Düsseldorf durchgeführt wurde (siehe EMMER/VOWE/WOLLING 2011) lässt sich das gut nachzeichnen: Während zwischen 2002 und 2005 noch Ausschöpfungsquoten zwischen 42 und 46 Prozent erreicht werden konnten, sanken diese bis 2008 auf zum Teil unter 30 Prozent (S. 70). Insgesamt ist die Datenlage hierzu zwar etwas unübersichtlich, da Ausschöpfungsquoten nicht immer explizit berichtet werden (siehe VON HERMANNI/ LEMCKE 2017), doch dürfte sich das angesichts einer stärkeren Standardisierung auch in diesem Bereich (siehe etwa GRAMLICH/HÄDER 2019) mit der Zeit ändern.

Wie groß das Problem niedriger Ausschöpfungsquoten tatsächlich ist, dazu liegen bereits einige Studien vor (etwa BLASIUS/REUBAND 1995, KOCH 1998, GROVES/PEYTCHEVA 2008). Ebenso wird versucht, die Gründe für den steigenden Nonresponse zu ergründen (siehe u.a. SCHNELL 1997, NELLER 2005, BAUR 2006, SCHNAUBER/DASCHMANN 2008). Dabei wurde zuletzt auch die Mobilfunktelefonie zunehmend in den Fokus genommen (HÄDER/HÄDER 2009, HÄDER/SCHNEIDERAT 2009, SCHLINZIG/SCHNEIDERAT 2009, FUCHS 2012, BUSSE/FUCHS 2015). In vergleichenden Studien zeigte sich dann, dass die Teilnahmebereitschaft über das Mobilfunknetz zunächst höher ausfiel als über das Festnetz. HÄDER ET AL. (2009) erklärten sich das mit einem „Überraschungsmoment“ (S. 73): „Da es in Deutschland bisher kaum Umfragen über das Mobilfunknetz gab“, so vermuten sie, „wird diese Art der Kontaktaufnahme und Befragung nicht sofort abgewehrt, sondern eher mit Erstaunen und positiver Resonanz quittiert" (ebd.). Spätere Studien deuten allerdings darauf hin, dass sich dieser Effekt mit der Zeit abnutzt und sich die Responseraten langsam angleichen (HÄDER/SAND 2019: 64-66).

\section{Werkstattbericht: Telefonbefragung zu „Digital Citizenship“}

Die oben vorgestellten Standards und Empfehlungen für die Ziehung von Telefonstichproben sollen im Folgenden anhand einer konkreten Telefonbefragung problemzentriert diskutiert und reflektiert werden. Als Fallbeispiel dient dazu die Befragungsstudie „Digital Citizenship - Politische Partizipation in Deutschland“, die vom Weizenbaum-Institut für die vernetzte Gesellschaft in Kooperation mit dem Institut für Publizistik- und Kommunikationswissenschaft der Freien Universität Berlin im Zeitraum von 9. September bis 30. November 2019 durchgeführt wurde. Im Fokus der Studie stand die Frage, welche Bürgernormen in der deutschen Bevölkerung bestehen und wie sie sich auf die politische Kommunikation und Partizipation im und außerhalb des Internets auswirken (für einen ersten Überblick über Anlage und Ergebnisse der Studie siehe SCHAETZ ET AL. 2020). Dazu wurden 1.298 Personen aus der deutschsprachigen Bevölkerung in Deutschland ab einem Alter von 16 Jahren befragt. Durchgeführt wurde die Befragung von 30 geschulten Interviewer*innen im Telefonstudio für computergestützte Telefonumfragen („Computer Assisted Telephone Interview“, CATI) am Institut für Publizistik- und Kommunikationswissenschaft der Freien Universität Berlin.

\subsection{Verhältnis von Festnetz- und Mobilfunkstichprobe}

Um ein Undercoverage insbesondere jüngerer Bevölkerungsteile, deren Auskünfte für die Befragungsstudie themenbedingt besonders relevant sind, möglichst zu vermeiden, wurde die Stichprobenziehung nach dem oben vorgestellten "Dual-Frame-Ansatz" (siehe Abschnitt 2.1) vorgenommen. Zurückgegriffen wurde dazu auf das Angebot der GESIS, die einen zweimal jährlich aktualisierten Auswahlrahmen für Festnetzstichproben nach dem Gabler-Häder- 
Design und einem gesonderten Auswahlrahmen für Mobilfunkstichproben bereitstellt. ${ }^{3}$ Die komplizierte Konstruktion der beiden Auswahlrahmen und die zufällige Ziehung der Rufnummern folgte damit den entsprechenden Standards und Empfehlungen. Zu entscheiden war in diesem Zusammenhang also nur, zu welchem Verhältnis Festnetz- und Mobilfunknummern in der Stichprobe gemischt werden sollten. Hier wird mit Verweis auf die Simulationsstudie der ADM (2012) ein Verhältnis von 60 zu 40, zumindest aber von 70 zu 30 empfohlen. Da diese Simulationsstudie allerdings im Jahr 2011 durchgeführt wurde und ihre Ergebnisse damit zum Zeitpunkt der anstehenden Befragung bereits acht Jahre alt waren, stellte sich die Frage, inwiefern diese Empfehlungen noch angemessen sind.

Tatsächlich weisen die Zahlen der BUNDESNETZAGENTUR (2019) zur Entwicklung der registrierten Mobilfunknummern in Deutschland einen starken Anstieg um über 20 Prozent von 114 Millionen im 4. Quartal 2011 auf 140 Millionen im 4. Quartal 2019 auf (siehe Abbildung 1). Zurückzuführen ist dies in erster Linie auf einen sprunghaften Anstieg zwischen den Jahren 2015 (114 Mio.) und 2016 (130 Mio.), hinter dem sich allerdings keine plötzliche Explosion der tatsächlichen Teilnehmerzahlen verbirgt, sondern der in erster Linie auf eine Umstellung der Zählweise bei Vodafone zurückzuführen ist. Zwar sind die Zahlen seither noch einmal um zehn Millionen Mobilfunknummern gestiegen, allerdings erscheinen signifikante Veränderungen des Mischverhältnisses von Festnetz- und Mobilfunknummern vor dem Hintergrund dieser Entwicklung noch nicht zwingend.

Anders sieht dies mit einem Blick auf die Entwicklung der „Mobile-onlys" in Deutschland aus. Das drohende Undercoverage dieser Bevölkerungsgruppe, die ausschließlich über den Mobilfunk zu erreichen ist, war damals das zentrale Argument für die Implementierung eines „Dual-Frame-Ansatzes“; insofern sollte die Entwicklung in den vergangenen Jahren eine Auskunft über ggf. notwendige Anpassungen des Mischungsverhältnisses von Festnetz- und Mobilfunknummern für eine Telefonstichprobe geben können. Zum Zeitpunkt der Simulationsstudie (19.09.-30.12.2011) lag der Anteil der „Mobile only“-Haushalte in Deutschland der Studie selbst zufolge bei 12,4 Prozent, während „Landline only“-Haushalte 18,6 Prozent ausmachten. 69 Prozent der Haushalte waren hingegen sowohl über Festnetz als auch über Mobilfunk („Dual use“) zu erreichen (siehe ADM 2012). Ähnlich fallen auch die Zahlen des Eurobarometers für das Jahr 2011 aus: Demnach liegen in Deutschland die Anteile der „Mobile only“-Haushalte bei elf Prozent, der „Landline only“-Haushalte bei 14 Prozent und der „Dual use“-Haushalte bei 75 Prozent (siehe EUROPÄISCHE KOMMISSION 2012: 14).

Seither haben sich die Verhältnisse diesbezüglich maßgeblich verändert: Im Eurobarometer Spezial zu diesem Thema aus dem Jahr 2017 lag der Anteil der „Mobile only“-Haushalte in Deutschland bei 14 Prozent, der "Landline only“-Haushalte bei 12 Prozent und der "Dual use“Haushalte nach einem zwischenzeitigen Anstieg auf 83 Prozent in 2013 nunmehr wieder bei 73 Prozent (EUROPÄISCHE KOMMISSION 2018: 32). Damit hat sich das das Anteilsverhältnis von "Mobile only“- zu „Landline only“-Haushalten seit 2011 im Grunde umgekehrt. Zwei Jahre zuvor war der Abstand mit 15 Prozent „Mobile only“- und nur 8 Prozent „Landline only“-Haushalten sogar noch deutlicher (EUROPÄISCHE KOMMISSION 2016: 43). In Anbetracht der sich für die kommenden Jahre abzeichnenden Entwicklung, der zufolge die Anteile der "Landline only“- und „Dual use“-Haushalte abnehmen, während der Anteil der „Mobile only“-Haushalte steigt (siehe Abbildung 3), spricht dies für eine baldige Wiederholung der Simulationsstudie von 2011 und ggf. eine Neuformulierung der Empfehlungen bezüglich des Verhältnisses von Festnetz- und Mobilfunknummern bei Stichprobenziehungen mit dualen Auswahlrahmen.

3 https://www.gesis.org/angebot/daten-erheben/ziehung-telefonstichproben 
Abbildung 3: Trends der Anteile von "Mobile onlys", "Landline onlys" und "Dual use" in deutschen Haushalten, 2005-2017

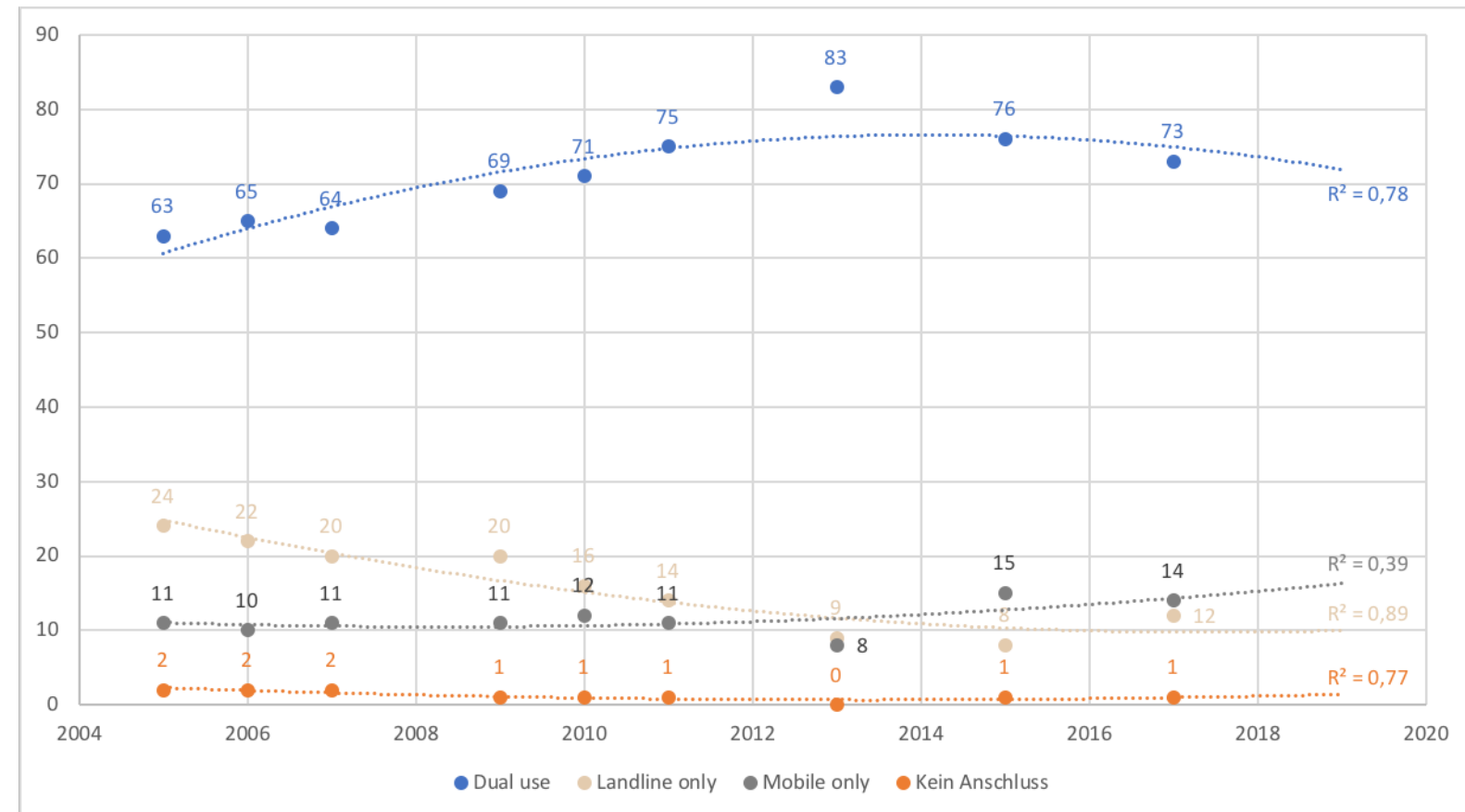

Anmerkung: Die abgetragenen Werte wurden jeweils für das Jahr der entsprechenden Befragung und nicht ihrer Veröffentlichung eingetragen (Datenquelle: Spezial Eurobarometer 2006-2018). Zur Veranschaulichung der Entwicklung über Zeit wurde eine polynomische Trendline ergänzt. Die $R^{2}$-Werte dienen als Bestimmtheitsmaß für die Zuverlässigkeit der jeweiligen Trendlinie. Der niedrige Wert bei „Mobile Only“ $\left(R^{2}=0,39\right)$ ist in erster Linie auf die starken Abweichungen im Jahr 2013 zurückzuführen. Ohne die Werte aus diesem Jahr steigt der Wert auf $R^{2}=0,80$ an. Auch der Wert für die „Dual use"-Trendlinie steigt dadurch nochmals erheblich auf $R^{2}=0,90$ an.

Neben der Entwicklung der prinzipiellen Erreichbarkeit über das Fest- oder das Mobilfunknetz kann auch ein Blick auf die tatsächliche Nutzung dieser beiden Netze bei der Einschätzung bezüglich einer möglicherweise notwendigen Überarbeitung der Empfehlungen helfen. So zeigen etwa die Daten des Verbands der Anbieter von Telekommunikations- und Mehrwertdiensten (VATM), dass die Nutzungszeiten der Festnetztelefonie (gemessen in abgehenden Sprachverbindungsminuten) bereits seit 2002 rückläufig sind - zunächst nur leicht, ab 2010 dann jedoch zunehmend stärker (siehe Abbildung 4). Im Gegensatz dazu sind die Nutzungszeiten der Mobilfunktelefonie seit 1998 kontinuierlich angestiegen, wobei der stärkste Zuwachs zwischen 2004 und 2010 zu verzeichnen ist. Diese gegenläufigen Entwicklungen führten 2018 schließlich dazu, dass die Nutzungszeiten der Mobilfunktelefonie die der Festnetztelefonie erstmalig übertrafen. Es kann in diesem Zusammenhang zumindest teilweise von einer Substitution der Festnetz- durch die Mobilfunktelefonie gesprochen werden (siehe HEINZ 2015). Gestützt werden diese Zahlen auch durch die BUNDESNETZAGENTUR (2019), die für die Zeit von 2010 bis 2018 eine ganz ähnliche Entwicklung aufzeigt (S. 48).

Für die Stichprobenziehung sind diese Entwicklungen aus zwei Gründen relevant: Zum einen deuten sie darauf hin, dass die Mobilfunktelefonie in der deutschen Bevölkerung über die vergangenen Jahre insgesamt wichtiger geworden - und zwar noch weitaus mehr als die reinen Anschlusszahlen vermuten lassen. Zum anderen wird deutlich, dass der große Anstieg der Mobilfunknutzung nicht auf eine Zunahme der "Mobile-onlys", sondern vor allem auf ein verändertes Telefonieverhalten in den „Dual use“-Haushalten zurückzuführen ist. So zeigt Abbildung 4, dass in dem Zeitraum zwischen 2004 und 2010, in der die Nutzungszeiten der Mobil- 
funktelefonie bislang am stärksten angestiegen sind, vor allem die Anteile der „Dual use“Haushalte merklich zugenommen haben, während die Anteile der "Mobile only“-Haushalte in demselben Zeitraum nahezu unverändert blieben (siehe Abbildung 3). Die angesprochene Substitution der Festnetz- durch die Mobilfunktelefonie hat also vor allem hier stattgefunden.

Abbildung 4: Abgehende Sprachverbindungsminuten im Festnetz und Mobilfunk in Deutschland, 1998-2018 (in Millionen Minuten pro Tag)

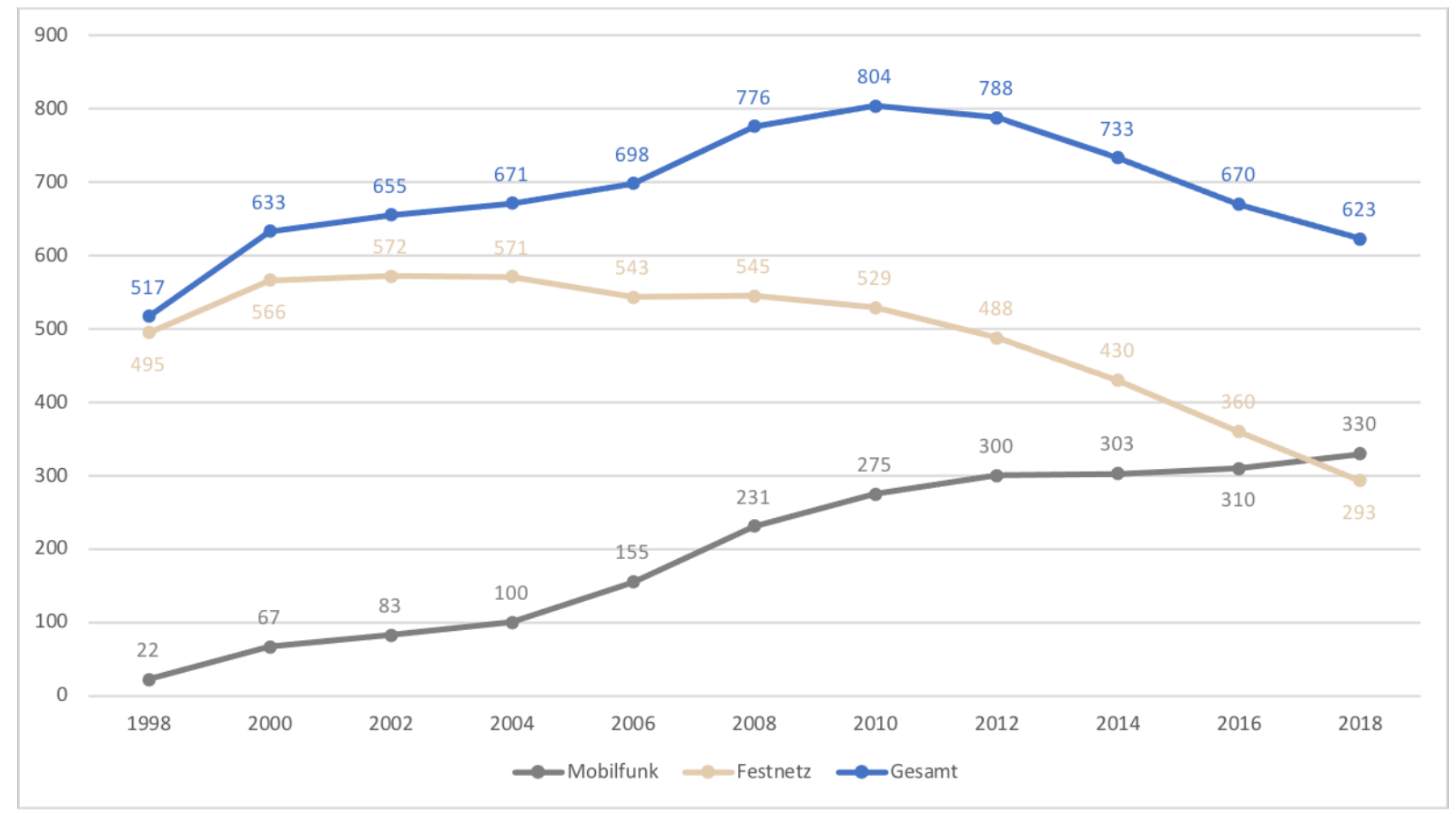

Anmerkung: Angaben ohne Roaming- und OTT-Verkehr. Datenquelle: DIALOG CONSULT/VATM (2019).

Da in diesem Zusammenhang nicht davon ausgegangen werden kann, dass sich die Veränderungen des Telefonieverhaltens in allen „Dual use“-Haushalten in gleicher Weise vollzogen haben, ist zu erwarten, dass es innerhalb dieser Gruppe gewichtige Unterschiede gibt. So ist laut SAND (2015) zu erwarten, „dass neben denjenigen Haushalten, die ausschließlich über einen der beiden Auswahlrahmen kontaktiert werden können, auch Haushalte existieren, die zwar in den Bereich der Dual-User fallen, jedoch besser über ein bestimmtes Telekommunikationsmedien erreichbar sind" (S. 143, siehe dazu auch HOOPS/MICHAEL 2015, SAND 2018). Für den Zweck einer präziseren Berechnung der Dual-Frame-Gewichtung unterteilt er die „Dual use“-Haushalte dementsprechend in drei Gruppen (siehe S. 144): erstens Haushalte, die "dominant das Telekommunikationsmedium Festnetz verwenden“ („Landline-Mainlys"), zweitens jene, die „keines der beiden Telekommunikationsmedien dominant verwenden“ („DualUser"), und drittens jene, die "dominant das Telekommunikationsmedium Mobilfunk verwenden“ („Mobile-Mainlys“). Eine solche differenziertere Betrachtung der „Dual use“-Haushalte gilt es im Falle einer Neuberechnung eines optimalen Verhältnisses von Festnetz- und Mobilfunknummern in dualen Auswahlraumen explizit zu berücksichtigen.

Vor diesem Hintergrund wurde sich im Zuge der Stichprobenziehung für die Telefonbefragung zu „Digital Citizenship” schließlich für einen Anteil von Mobilfunknummern an der Gesamtstichprobe von 50 Prozent entschieden. Obwohl damit über die Empfehlung der ADM (2012) hinausgegangen wurde, liegt dieser Anteil noch immer in dem von ihr definierten „vertretbaren Rahmen“ (S. 31) zwischen 20 und 60 Prozent. Gleichwohl bleibt diese Festlegung 
spekulativ und deshalb stichprobentheoretisch problematisch, so lange keine aktuelleren Zahlen vorliegen. Ein Festhalten an den bisherigen Empfehlungen ist aus den oben dargestellten Gründen allerdings mit ähnlichen Problemen behaftet. Aus diesem Grund bedarf es in naher Zukunft dringend einer Neuauflage der ADM-Simulationsstudie und einer Aktualisierung der Empfehlungen, auch wenn entsprechende Anpassungen der dualen Auswahlrahmen in der Folge mit merklich erhöhten Kosten verbunden sein dürften. Dafür muss sich allerdings auch die Perspektive auf die Notwendigkeit von „Dual-Frame“-Ansätzen grundlegend ändern: Angesichts der steigenden Anteile der "Mobile only“-Haushalte sowie der insgesamt zunehmenden Bedeutung der Mobilfunktelefonie auch in den „Dual use“-Haushalten ist davon auszugehen, dass sich der Mobilfunk in Deutschland bereits als Standard durchgesetzt hat und das Festnetz nur noch eine Nebenrolle spielt.

\subsection{Durchführung des HLR-Lookup}

Nach Ziehung der Festnetz- und Mobilfunknummern galt es nun, letztere durch das von HÄDER und SAND (2019) empfohlene HLR-Lookup prüfen zu lassen und zu bereinigen. Im Vorfeld der Befragung wurden dazu zunächst zwei verschiedene Anbieter für HLR-Lookups kontaktiert: zum einen Velocity Made Good Ltd. ${ }^{4}$, das als MyCoolSMS bei der Evaluationsstudie von SAND (2017) hinsichtlich der Validität der Lookup-Ergebnisse sehr gut abgeschnitten hat; zum anderen die Bulktrade GmbH. ${ }^{5}$ Auf Anfrage teilte Bulktrade mit, dass zahlreiche Netzbetreiber zu jener Zeit versuchen würden, „alle Provider zu blockieren, die solche HLR stellen“. Dieses Problem wurde von Velocity Made Good bestätigt, die ebenfalls auf Anfrage mitteilten: „Telekom indeed does not work currently, Telefonica and other networks should be okay". Es scheint sich dabei um ein neueres Problem zu handeln, denn weder ist davon in der Evaluationsstudie von SAND (2017) die Rede, noch in dem Beitrag von HÄDER und SAND (2019). Aus diesem Grund wurde das Lookup-Verfahren zuerst einmal anhand der für den Pretest gezogenen Mobilfunkstichprobe getestet, um zu prüfen, ob eine Anwendung auf die gesamte Mobilfunkstichprobe der Hauptbefragung überhaupt (noch) sinnvoll ist. Aufgrund der geringeren Kosten sowie der positiven Evaluation durch SAND (2017) wurde dazu auf den Dienst HLRLookups.com von Velocity Made Good zurückgegriffen.

Die Pretest-Stichprobe umfasste 2.398 Rufnummern, von denen 1.081 Telefónica 02, 643 Vodafone und 674 T-Mobile zugeordnet werden konnten. Letztere wurden aufgrund des Hinweises durch Velocity Made Good von der Test-Prüfung ausgeschlossen. Für die übrigen 1.724 Rufnummern wurde drei Tage vor Beginn des Pretests, am 24. August 2019, das HLR-Lookup durchgeführt. Der Test des Verfahrens bestand dann darin, die Mobilfunkstichprobe nach Durchführung dieses Lookups beizubehalten und dessen Ergebnisse - analog zum Vorgehen bei SAND (2017) - durch die im Pretest ermittelten Kontaktergebnisse zu validieren.

Der HLR-Lookup kam dabei zunächst zu den folgenden Ergebnissen: Für 369 Rufnummern wurde der Status „connected“ (gültig und mit dem Netzwerk verbunden) ausgegeben, für 220 Nummern „absent“ (gültig, jedoch nicht mit dem Netzwerk verbunden), elf Rufnummern waren „invalid“ (ungültig), bei 444 Rufnummern war der Status nicht zu bestimmen („undetermined“) und für 680 Nummern lautete er „unknown“ (im Netzwerk unbekannt bzw. nicht vergeben). Nach SAND (2017) können nur letztere aus der Stichprobe aussortiert werden, ohne dass es dabei zu einer falsch-negativen Selektion kommt. Der Abgleich mit den Kontaktergebnissen aus der anschließenden Pretest-Befragung zeigt allerdings, dass alle diese 680 Rufnum-

$4 \quad$ https://www.hlr-lookups.com

5 https://www.bulktrade.de 
mern tatsächlich auch von der Dialer-Software (Voxco) als „nicht vergeben“ erkannt wurden, während die anderen Lookup-Ergebnisse - wie schon bei SAND (2017) - durch die Kontaktergebnisse der Befragung nicht so eindeutig validiert werden konnten (siehe Tabelle 1).

Tabelle 1: Gegenüberstellung der Ergebnisse von HLR-Lookup und Pretest-Befragung

\begin{tabular}{lrrr}
\hline HLR-Lookup-Status & \multicolumn{3}{c}{ Kontaktergebnisse im CATI-Pretest } \\
\cline { 2 - 4 } & RN nicht vergeben & andere Ergebnisse & Gesamt \\
\hline connected & $29(20 \%)$ & $340(80 \%)$ & $369(100 \%)$ \\
absent & $45(8 \%)$ & $175(92 \%)$ & $220(100 \%)$ \\
invalid & $0(0 \%)$ & $11(100 \%)$ & $11(100 \%)$ \\
undetermined & $389(88 \%)$ & $55(12 \%)$ & $444(100 \%)$ \\
unknown & $680(100 \%)$ & $0(0 \%)$ & $680(100 \%)$ \\
\hline Gesamt & $1.143(66 \%)$ & $581(44 \%)$ & $1.724(100 \%)$ \\
\hline
\end{tabular}

Insofern ist das Verfahren zwar nach wie vor zuverlässig, allerdings werden damit nur (noch) solche Rufnummern aussortiert, die im Zuge des Predialings später ohnehin automatisch und kostenneutral aussortiert werden. Für die Bereinigung der Mobilfunkstichprobe bedeutet dies, dass der HLR-Lookup - zumindest bei der Nutzung von Telefonanlagen mit automatischem Predialing - faktisch keinen Mehrwert (mehr) bringt. Denn das eigentliche Ziel einer Verminderung der Kontakte mit Anrufbeantwortern wird damit nicht mehr erreicht. Aus diesem Grund wurde für die Mobilfunkstichprobe der Hauptbefragung schließlich auch kein HLRLookup mehr durchgeführt.

\subsection{Ausschöpfungsquote}

Für die Hauptbefragung zu „Digital Citizenship“ wurden die Festnetz- und die Mobilfunkstichprobe schließlich zu einer zufällig sortierten Stichprobe zusammengelegt und abtelefoniert. Im Falle der Festnetznummern wurden die zu befragenden Zielpersonen dabei über die sogenannte "Last-Birthday-Methode" bestimmt, da über Festnetznummern häufig mehrere Personen in einem gemeinsamen Haushalt erreichbar sind und die Inklusionswahrscheinlichkeit deshalb zugunsten derjenigen verzerrt würde, die in diesen Haushalten Anrufe wahrscheinlicher entgegennehmen (siehe MAURER 2005). Bei den Mobilfunknummern wurde hingegen die antwortende Person ohne ein weiteres Auswahlverfahren direkt befragt, "da davon ausgegangen werden kann, dass Mobiltelefonnummern in der überwiegenden Mehrheit Personen und nicht Haushalten zugeordnet werden“ (HÄDER/SAND 2019: 72-73). Alle Rufnummern wurden gemäß der „Richtlinien für telefonische Befragungen“ der ADM (2016) mit einem Maximum von zehn Erstkontaktversuchen angerufen, wobei nicht mehr als zwei Versuche pro Tag vorgenommen und die Anrufzeiten sowohl über verschiedene Wochentage als auch verschiedene Tageszeiten gestreut wurden. Eine Ausnahme von dem Maximum der zehn Erstkontaktversuche bildeten lediglich jene Fälle, bei denen die ersten fünf Anrufe zu einem Anrufbeantworter führten. Sie wurden mit einem entsprechenden Kontaktergebnis aussortiert und nicht weiter angerufen, um das Interviewpersonal nicht weiter zu belasten (siehe Abschnitt 2.1).

Zur Berechnung der Ausschöpfungsquoten im Nachgang der Befragung wurden die finalen Kontaktergebnisse in die vier von der AAPOR (2016) definierten Kategorien sortiert: Neben den 1.298 geführten Interviews (1), waren dies 11.199 ,non-respondents' (2), 20.603 unklare 
Fälle (3) und 130.702 stichprobenneutrale Ausfälle (4). ${ }^{6}$ Nach den Berechnungsvorgaben der AAPOR (2016) liegt die Ausschöpfungsquote demzufolge zwischen einem Minimum von RR1 = 3,92 Prozent und einem Maximum von RR5 $=10,39$ Prozent. Teilt man die unklaren Fälle dabei auf in (a) eine Gruppe von Fällen mit wechselnden Kontaktergebnissen, und (b) in eine Gruppe von Fällen, bei denen sich jeweils ein bestimmtes Kontaktergebnis immer wiederholt hat (z.B. 10x besetzt, 10x Freizeichen oder 5x Anrufbeantworter), ergibt sich eine Verteilung der stichprobenrelevanten Rufnummern wie sie in Abbildung 5 abgetragen ist. Hier wird deutlich, dass mit 13.228 Rufnummern über 60 Prozent der unklaren Fälle in die Gruppe (b) mit sich wiederholenden Kontaktergebnissen fallen. Die größte Teilgruppe umfasst dabei 8.500 Fälle, bei denen in allen zehn Malen ein Freizeichen ertönte, wobei mit 6.344 Fällen nahezu drei Viertel dieser Fälle auf das Festnetz entfallen. Die zweitgrößte Teilgruppe umfasst 4.450 Fälle, bei denen bei den ersten fünf Anrufen ein Anrufbeantworter erreicht wurde. Wie zu erwarten war, entfallen davon mit 4.114 Fällen über 90 Prozent auf das Mobilfunknetz. Bei den restlichen 278 unklaren Fällen mit repetitiven Kontaktergebnissen waren die Rufnummern bei allen zehn Anrufen besetzt. Mit 243 Fällen macht hier wiederum das Festnetz einen Anteil von fast 90 Prozent aus. Es zeigt sich also, dass das Problem mit den unklaren Fällen bei Mobilfunk und Festnetz unterschiedlich gelagert ist: Während wir es beim Mobilfunk mit dem bekannten Problem der vielen Anrufbeantworter zu tun haben (siehe Abschnitt 2.1), besteht das Problem im Festnetz vor allem in jenen Fällen, bei denen - selbst über mehrere Wochen hinweg - immer nur ein Freizeichen zu hören ist.

\section{Abbildung 5: Kontaktergebnisse der stichprobenrelevanten Fälle}

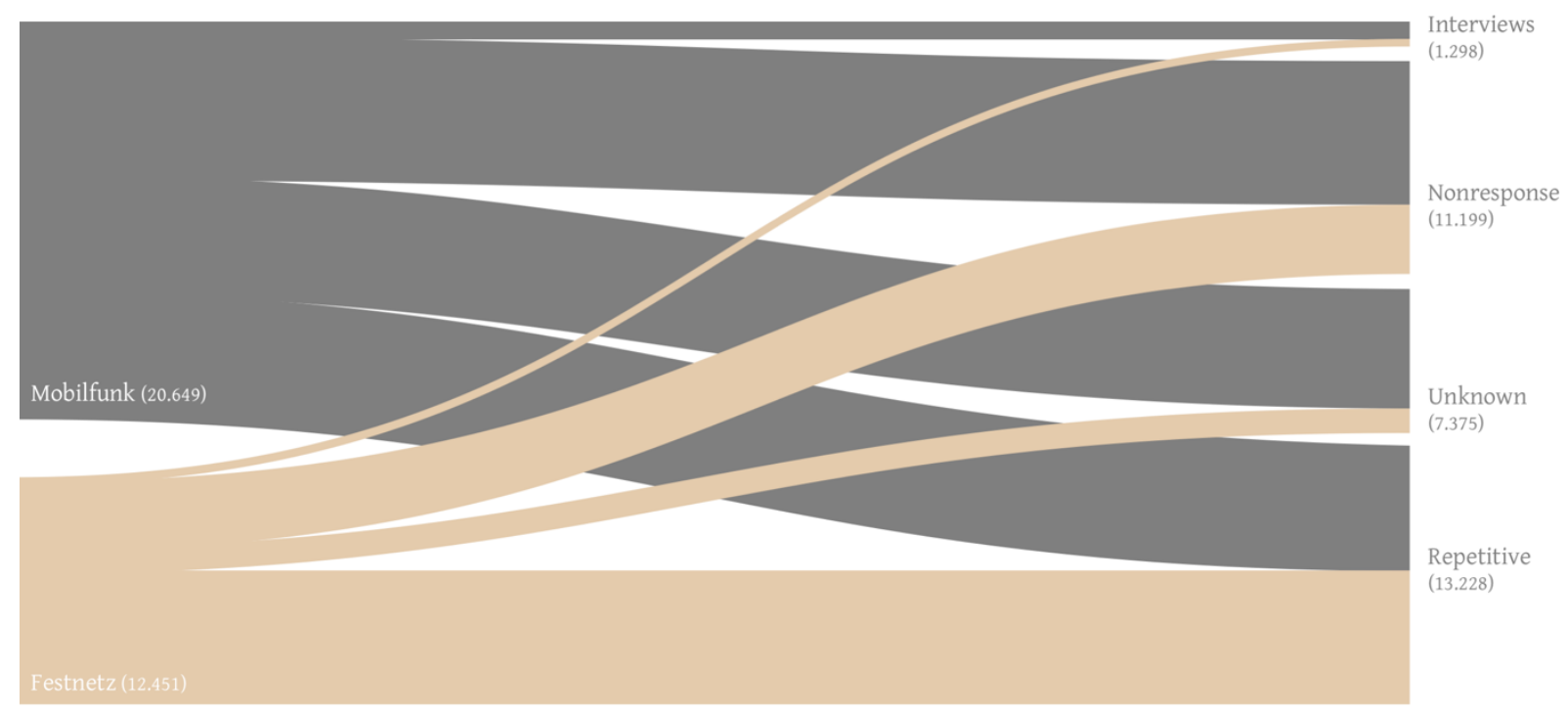

Behandelt man nun alle 13.228 Rufnummern mit repetitiven Kontaktergebnissen als stichprobenneutral und die übrigen 7.375 unklaren Fälle als stichprobenrelevant, ergibt sich eine Ausschöpfungsquote von RR3 $=6,53$. Ein Ausschluss der unklaren Fälle mit repetitiven Kontaktergebnissen führt also fast zu einer Verdoppelung der Responserate. Insofern wäre es wichtig,

6 Die hohe Anzahl der stichprobenneutralen Ausfälle (Kategorie 4) ist auf einen Fehler bei der Zusammenstellung der Stichprobe zurückzuführen, bei der die Festnetznummern versehentlich um weitere Ziffern ergänzt wurden. Dadurch wurden viele Rufnummern ungültig, weshalb die Stichprobe so stark aufgestockt werden musste. Dieser Fehler wurde erst im Nachgang der Befragung erkannt. Die außergewöhnlich hohe Anzahl der stichprobenneutralen Ausfälle ist deshalb nicht aussagekräftig. 
für die Zukunft einen einheitlichen und verlässlichen Weg zu finden, mit diesen Fällen umzugehen. In Anbetracht der beschriebenen Entwicklung der Ausschöpfungsquoten in den vergangenen Jahren (siehe Abschnitt 2.3) sind die hier ausgewiesenen niedrigen Werte darüber hinaus nicht völlig überraschend; gleichwohl stellt sich die Frage, ob sich die Abnahme der Teilnahmebereitschaft auch in Zukunft noch fortsetzen wird oder ob sie sich langsam auf diesem Wert eingependelt hat. Hier gilt es, die weitere Entwicklung auch in Zukunft genau zu beobachten, die ausschlaggebenden Gründe dafür zu ermitteln und ggf. mit Anpassungen in der Stichprobenziehung darauf zu reagieren.

Für Optimismus in dieser Frage sorgten vor einigen Jahren noch die aufkommenden DualFrame-Ansätze bzw. die stärkere Einbeziehung von Mobilfunknummern in die Telefonstichproben. Denn die Teilnahmebereitschaft war bei Anrufen auf Mobiltelefonen zu Beginn noch weitaus höher als bei Festnetz. HÄDER ET AL. (2009) äußerten dabei die Hoffnung, „dass es sich hierbei nicht um einen kurz befristeten Effekt handelt" (S. 73). In der Folgezeit glichen sich die Responseraten im Mobilfunk allerdings immer weiter an jene im Festnetz an (HÄDER/ SAND 2019: 64-66). Und tatsächlich bestätigen auch die Responseraten der „Digital Citizenship“-Befragung diesen Trend. Zwar liegen die minimale und die maximale Responserate im Mobilfunk noch immer über jenen, die über das Festnetz erzielt werden konnten, doch ist der Unterschied hier nicht mehr allzu groß (siehe Tabelle 2). Ausschlaggebend ist dabei vor allem die erheblich höhere Anzahl unklarer, nicht-repetitiver Fälle, die schwer zu interpretieren sind und die Ausschöpfungsquote im Mobilfunk nach unten drücken. Das zeigt sich auch im Vergleich der geschätzten Responseraten (RR3), bei denen alle unklaren Fälle mit repetitiven Kontaktergebnissen aussortiert wurden. Hier liegt die Ausschöpfung für das Festnetz sogar höher als die für den Mobilfunk.

Tabelle 2: Responseraten der „Digital Citizenship“-Befragung nach AAPOR (2016)

\begin{tabular}{lrrr}
\hline Auswahlrahmen & \multicolumn{3}{c}{ Responseraten } \\
\cline { 2 - 4 } & RR1 (min) & RR3 & RR5 (max) \\
\hline Festnetz & 3,19 & 7,18 & 9,50 \\
Mobilfunk & 4,36 & 6,28 & 10,83 \\
\hline Gesamt & 3,92 & 6,53 & 10,39 \\
\hline
\end{tabular}

\section{Schlussfolgerungen und Ausblick}

Aus der Gegenüberstellung der geläufigen Standards und Empfehlungen für Telefonumfragen in Deutschland einerseits und den hier berichteten Erfahrungen aus der jüngeren Befragungspraxis andererseits lassen sich drei zentrale Schlussfolgerungen für die Zukunft dieses Forschungsbereiches ziehen. Im Bereich der Stichprobenziehung ist erstens fraglich geworden, wie geeignet die auf der ADM-Simulationsstudie von 2011 basierenden Empfehlungen bezüglich des Verhältnisses von Festnetz- und Mobilfunknummern bei Stichprobenziehungen mit dualen Auswahlrahmen noch sind. Sowohl die Entwicklung der "Landline only"-, "Mobile only“- und „Dual use“-Haushalte in der deutschen Bevölkerung als auch die Trends in der konkreten Nutzung von Festnetz und Mobilfunk deuten darauf hin, dass sich seither einiges verändert hat, das für entsprechende Empfehlungen zur Stichprobenziehung relevant ist. Besondere Berücksichtigung bei der Neuberechnung eines optimalen Verhältnisses von Festnetz- 
und Mobilfunknummern verlangt dabei insbesondere die Nutzung von Festnetz und Mobilfunk als eine Voraussetzung für telefonische Erreichbarkeit über die beiden Netze. Hilfreiche Ansatzpunkte finden sich dazu vor allem bei SAND $(2015,2018)$.

Im Bereich der Datenbereinigung gilt es zweitens in absehbarer Zeit immer wieder erneut zu prüfen, inwieweit das HLR-Lookup für seinen ursprünglichen Zweck der Aussortierung nicht geschalteter Mobilfunknummern noch nützlich sein kann. Die Prüfung der Mobilfunkstichprobe für den Pretest der „Digital Citizenship“-Befragung hat gezeigt, dass dieses Verfahren zwar nach wie vor zuverlässig funktioniert, seine Ergebnisse allerdings auch von automatischen Predialern schnell und kostenneutral repliziert werden können. Wenn es bei dieser eingeschränkten Funktionalität bleiben sollte, würde ein HLR-Lookup nur noch für manuell durchgeführte Erhebungen einen tatsächlichen Mehrwert bringen. Auf Nachfrage ein Jahr nach dem HLR-Lookup zum Pretest der Befragungsstudie erklärte Velocity Made Good allerdings, dass „Germany and T-Mobile work fine again“. Insofern sollte der HLR-Lookup als Möglichkeit zur Bereinigung der Mobilfunkstichprobe nicht vorschnell aufgegeben werden. Stattdessen wäre es insbesondere bei umfangreichen Befragungsstudien wichtig, dass Verfahren - analog zu dem oben geschilderten Vorgehen -immer wieder anhand kleinerer Substichproben zu testen und die Ergebnisse dieser Tests mit zu berichten.

Drittens gilt es, sich in Zukunft noch stärker dem Problem unklarer Fälle bei der Berechnung der Stichprobenausschöpfung zu widmen und hier ggf. neue Standards zu entwickeln. Im Fokus sollten dabei diejenigen Fälle mit repetitiven Kontaktergebnissen - insbesondere den zahlreichen Anrufbeantwortern im Mobilfunknetz - stehen. Diese Fälle belasten nicht nur die Interviewer*innen, sondern wirken sich auch erheblich auf die Responseraten aus. Beispielhaft wurden in der „Digital Citizenship“-Befragung alle Fälle aussortiert, bei denen die ersten fünf Anrufe zu Anrufbeantwortern führten. Ein solches Vorgehen ist derzeit noch spekulativ, könnte sich jedoch etablieren, wenn mehr über diese Fälle bekannt wäre. So bietet es sich etwa an, solche Fälle nach unterschiedlich großen Zeitabständen erneut zu kontaktieren, um besser nachvollziehen zu können, hinter wie vielen dieser Fälle sich möglicherweise doch geschaltete Anschlüsse verbergen. Eine solche Überprüfung der unklaren Fälle wird vor allem dann umso wichtiger, wenn die Anteile der Mobilfunknummern in dualen Auswahlrahmen steigen und das HLR-Lookup als Verfahren zur Bereinigung der Mobilfunkstichprobe ausfällt.

\section{Literaturverzeichnis}

AAPOR - AMERICAN ASSOCIATION OF PUBLIC OPINION RESEARCH: New Considerations for

Survey Researchers When Planning and Conducting RDD Telephone Surveys in the U.S. With Respondents Reached via Cell Phone Numbers (AAPOR Cell Phone Task Force Report). 2010. https://www.a-

apor.org/AAPOR_Main/media/MainSiteFiles/2010AAPORCellPhoneTFReport.pdf AAPOR - AMERICAN ASSOCIATION OF PUBLIC OPINION RESEARCH: Standard Definitions. Final

Dispositions of Case Codes and Outcome Rates for Surveys. 2016. https://www.aapor.org/AAPOR_Main/media/publications/Standard-Definitions20169theditionfinal.pdf ADM - ARBEITSKREIS DEUTSCHER MARKT- UND SOZIALFORSCHUNGSINSTITUTE: ADM-

Forschungsprojekt „Dual-Frame-Ansätze“ 2011/2012. Forschungsbericht. 2012. https://www.adm-ev.de/wp-content/uploads/2018/07/Forschungsbericht-Dual-FrameAnsätze.pdf 
ADM - ARBEITSKREIS DEUTSCHER MARKT- UND SOZIALFORSCHUNGSINSTITUTE: StichprobenVerfahren in der Umfrageforschung: Eine Darstellung für die Praxis (2., aktualisierte und erweitere Auflage). Wiesbaden [Springer VS] 2014.

ADM - ARBEITSKREIS DEUTSCHER MARKT- UND SOZIALFORSCHUNGSINSTITUTE: Richtlinie für telefonische Befragungen. 2016. https://www.adm-ev.de/wp-content/uploads/2018/07/RL-Telefonbefragung.pdf

ADM - ARBEITSKREIS DEUTSCHER MARKT- UND SOZIALFORSCHUNGSINSTITUTE: Offener Brief des ADM - Repräsentativität von Umfragen und Kommunikation in den Medien. 22.10.2018. https://www.adm-ev.de/2018/10/22/offener-brief_repraesentativitaet/ AUST, F.; SCHRÖDER, H.: Sinkende Stichprobenausschöpfung in der Umfrageforschung - ein Bericht aus der Praxis. In WEICHBOLD, M.; BACHER, J.; WOLF, C. (Hrsg.): Umfrageforschung: Herausforderungen und Grenzen. Wiesbaden [VS Verlag] 2009, S. 195-212.

BAUR, N.: Ausfallgründe bei zufallsgenerierten Telefonstichproben am Beispiel des GablerHäder-Designs. In FAULBAUM, F.; WOLF, C. (Hrsg.): Stichprobenqualität in Bevölkerungsumfragen. Bonn [Informationszentrum Sozialwissenschaften] 2006, S. 159-183.

BLASIUS, J.; REUBAND, K.-H.: Telefoninterviews in der empirischen Sozialforschung: Ausschöpfungsquoten und Antwortqualität. In: ZA-Informationen, 37, 1995, S. 64-87.

BOWLEY, A. L.: The Application of Sampling to Economic and Sociological Problems. In: Journal of the American Statistical Association, 31(195), 1936, S. 474-480.

BOUNCKEN, R.: Gutachten zur Aussagefähigkeit von webbasierten Datenerhebungen und analysen. Beurteilung und gute Handlungspraxis am Beispiel von Civey. 2018. https://civey.com/ueber-civey/unsere-methode/artikel/gutachten-zur-aussagefaehigkeit-von-webbasierten-datenerhebungen-und

BUNDESNETZAGENTUR: Tätigkeitsbericht Telekommunikation 2018/2019. 2019. https://www.bundesnetzagentur.de/SharedDocs/Mediathek/Taetigkeitsberichte/2019/TK_20182019.pdf

BUNDESNETZAGENTUR: Teilnehmerentwicklung im Mobilfunk. 2020. https://www.bundesnetzagen-

tur.de/DE/Sachgebiete/Telekommunikation/Unternehmen_Institutionen/Marktbeobacht ung/Deutschland/Mobilfunkteilnehmer/Mobilfunkteilnehmer_node.html

BUSSE, B.; FUCHS, M.: The components of landline telephone survey coverage bias. The relative importance of no-phone and mobile-only populations. In: Quality \& Quantity, 46(4), 2012, S. 1209-1225. doi:10.1007/s11135-011-9431-3

BUSSE, B.; FUCHS, M.: Telephone Surveys Using Mobile Phones. In ENGEL, U.; JANN, B.; LYNN, P.; SCHERPENZEEL, A.; STURGIS, P. (Hrsg.): Improving Survey Methods: Lessons from Recent Research. New York [Routledge] 2015, S. 51-66.

CAllegaro, M.; AYhAN, Ö.; GABler, S.; HÄDER, S.; VILlaR, A.: Combining landline and mobile phone samples: a dual frame approach (GESIS-Working Papers, 2011/13). Köln [GESIS - Leibniz-Institut für Sozialwissenschaften] 2011.

CIVEY: Die Statistische Methodik von Civey Eine Einordnung im Kontext gegenwärtiger Debatten über das Für und Wider internetbasierter nicht-probabilistischer Stichprobenziehung. Whitepaper. 2020. https://civey.com/ueber-civey/unsere-methode

CURTIN, R.; PRESSER, S.; SINGER, E.: Changes in telephone survey nonresponse over the past quarter century. In: Public Opinion Quarterly, 69(1), 2005, S. 87-98. doi:10.1093/poq/nfi002 
DEUTSCHMANN, M.; HÄDER, S.: Nicht-Eingetragene in CATI-Surveys. In GABLER, S.; HÄDER, S. (Hrsg.): Telefonstichproben: Methodische Innovationen und Anwendungen in Deutschland. Münster [Waxmann] 2002, S. 68-84.

DIALOG CONSULT; VATM: 21. TK-Marktanalyse Deutschland 2019. Ergebnisse einer Befragung der Mitgliedsunternehmen im Verband der Anbieter von Telekommunikations- und Mehrwertdiensten e. V. im dritten Quartal 2019. https://www.vatm.de/wp-content/uploads/2019/10/VATM_TK-Marktstudie_2019_091019.pdf

EMMER, M.; VOWE, G.; WOLLING, J.: Bürger Online: Die Entwicklung der politischen OnlineKommunikation in Deutschland. Konstanz [UVK] 2011.

EUROPÄISCHE KOMMISSION: Spezial Eurobarometer 381: Haushaltsumfrage zur E-Kommunikation - Bericht. 2012.

EUROPÄISCHE KOMMISSION: Spezial Eurobarometer 438: Elektronische Kommunikation und digitaler Binnenmarkt - Bericht. 2016.

EUROPÄISCHE KOMMISSION: Spezial-Eurobarometer 462: Elektronische Kommunikation und digitaler Binnenmarkt - Bericht. 2018.

FOLLMER, T.; SMID, M.: Nichteingetragene Telefonnummern: Ergebnisse eines Methodentests. In GABLER, S.; HÄDER, S.; HOFFMEYER-ZLOTNIK, J.H.P. (Hrsg.): Telefonstichproben in Deutschland. Wiesbaden [VS Verlag] 1998, S. 43-57.

FREY, J. H.; KUNZ, G.; LÜSCHEN, G.: Telefonumfragen in der Marktforschung: Methoden, Techniken, Befragungspraxis. Opladen [Westdeutscher Verlag] 1990.

FRIES, S.: Methodenstreit der Meinungsforschung: Was ist repräsentativ? Deutschlandfunk. 03.12.2018. https://www.deutschlandfunk.de/methodenstreit-der-meinungsforschungwas-ist-repraesentativ.724.de.html?dram:article_id=434924

FUCHS, M.: Der Einsatz von Mobiltelefonen in der Umfrageforschung Methoden zur Verbesserung der Datenqualität. In FAULBAUM, F.; STAHL, M.; WIEGAND, E. (Hrsg.): Qualitätssicherung in der Umfrageforschung. Wiesbaden [Springer VS] 2012, S. 51-73.

GABLER, S.; AYHAN, Ö.: Gewichtung bei Erhebungen im Festnetz und über Mobilfunk: Ein Dual-Frame-Ansatz. In GABLER, S.; HÄDER, S. (Hrsg.), Mobilfunktelefonie - Eine Herausforderung für die Umfrageforschung. Mannheim [GESIS-ZUMA] 2007, S. 39-46.

GABLER, S.; HÄDER, S.: Überlegungen zu einem Stichprobendesign für Telefonumfragen in Deutschland. In: ZUMA-Nachrichten, 41, 1997, S. 7-18.

GABLER, S.; HÄDER, S.: Probleme bei der Anwendung von RLD-Verfahren. In GABLER, S.; HÄDER, S.; HOFFMEYER-ZLOTNIK, J.H.P. (Hrsg.): Telefonstichproben in Deutschland. Wiesbaden [VS Verlag] 1998, S. 58-68.

GABLER, S.; HÄDER, S.: Erfahrungen beim Aufbau eines Auswahlrahmens für Telefonstichproben in Deutschland. In: ZUMA-Nachrichten, 43, 1999, S. 45-61.

GABLER, S.; HÄDER, S.: Mitteilung über die Gründung der Arbeitsgruppe Mobilsample. In: ZUMA-Nachrichten, 56, 2005, S. 111-116.

GABLER, S.; HÄDER, S.: Die Kombination von Mobilfunk- und Festnetzstichproben in Deutschland. In WEICHBOLD, M.; BACHER, J.; WOLF, C. (Hrsg.): Umfrageforschung: Herausforderungen und Grenzen. Wiesbaden [VS Verlag] 2009, S. 239-252.

GABLER, S.; HÄDER, S.: Stichproben in der Theorie (GESIS Survey Guidelines). Mannheim [GESIS - Leibniz-Institut für Sozialwissenschaften] 2015. doi:10.15465/gesis-sg_009

GABLER, S.; HÄDER, S.: Repräsentativität: Versuch einer Begriffsbestimmung. In HÄDER, S.; HÄDER, M.; SCHMICH, P. (Hrsg.): Telefonumfragen in Deutschland. Wiesbaden [Springer VS] 2019, S. 81-112. 
GABLER, S.; SCHÜRLE, J.: Zur Stabilität des Gabler-Häder-Auswahlrahmens. In GABLER, S.; HÄDER, S. (Hrsg.): Telefonstichproben: Methodische Innovationen und Anwendungen in Deutschland. Münster [Waxmann] 2002, S. 59-67.

GALLUP, G.: A guide to public opinion polls. Princeton [Princeton University Press] 1944.

GLEMSER, A.: ADM-Telefonstichproben in der Praxis. In GABLER, S.; HÄDER, S. (Hrsg.): Telefonstichproben: Methodische Innovationen und Anwendungen in Deutschland. Münster [Waxmann] 2002, S. 46-58.

GLEMSER, A.: Mobilfunknutzung in Deutschland: Eine Herausforderung für die Stichprobenbildung in der Markt- und Sozialforschung. In GABLER, S.; HÄDER, S. (Hrsg.): Mobilfunktelefonie - eine Herausforderung für die Umfrageforschung. Mannheim [GESIS-ZUMA] 2007, S. 7-23.

GLEMSER, A.; MEIER, G.; HECKEL, C.: Dual-Frame: Stichprobendesign für CATI-Befragungen im mobilen Zeitalter. In ADM - ARBEITSKREIS DEUTSCHER MARKT- UND

SOZIALFORSCHUNGSINSTITUTE (Hrsg.): Stichproben-Verfahren in der Umfrageforschung: Eine Darstellung für die Praxis (2., aktualisierte und erweitere Auflage). Wiesbaden [Springer VS] 2014, S. 167-190.

GUteRBOCK, T. M.; LAVRAKAS, P. J.; TOMPSON, T. N.; ZUWALLACK, R.: Cost and Productivity Ratios in Dual-Frame RDD Telephone Surveys. In: Survey Practice, 4(2), 2011, S. 1-7. doi:10.29115/SP-2011-0008

GRAMLICH, T.; LIEBAU, M.; SCHUNTER, J.: Die Berechnung von Responseraten. In HÄDER, S.; HÄDER, M.; SCHMICH, P. (Hrsg.): Telefonumfragen in Deutschland. Wiesbaden [Springer VS] 2019, S. 293-347.

GRAMLICH, T.; HÄDER, S.: Methoden- und Feldberichte. In HÄDER, S.; HÄDER, M.; SCHMICH, P. (Hrsg.): Telefonumfragen in Deutschland. Wiesbaden [Springer VS] 2019, S. 425-434.

GROVES, R. M.: Nonresponse rates and nonresponse bias in household surveys. In: Public $O$ pinion Quarterly, 70(5), 2006, S. 646-675. doi:10.1093/poq/nfl033

GROVES, R.M.; PEYTCHEVA, E.: The impact of nonresponse rates on nonresponse bias: A meta-analysis. Public Opinion Quarterly, 72(2), 2008, S. 167-189.

doi:10.1093/poq/nfn011

GRUND, M.: Mobilfunkteilnehmer - ein Repräsentanzproblem. In GABLER, S.; HÄDER, S. (Hrsg.): Telefonstichproben: Methodische Innovationen und Anwendungen in Deutschland. Münster [Waxmann] 2002, S. 111-120.

HÄDER, S.: Auswahlverfahren bei Telefonumfragen. ZUMA-Arbeitsbericht Nr. 94/03. 1994. HÄDER, S.: Wer sind die „Nonpubs“? Zum Problem anonymer Anschlüsse bei Telefonumfragen. In: ZUMA-Nachrichten, 39, 1996, S. 45-68.

HÄDER, S.: Telefonstichproben (ZUMA How-to-Reihe, Nr. 6). Mannheim [Zentrum für Umfragen, Methoden und Analysen] 2000.

HÄDER, S.: Stichproben in der Praxis (GESIS Survey Guidelines). Mannheim [GESIS - LeibnizInstitut für Sozialwissenschaften] 2015. doi:10.15465/gesis-sg_014

HÄDER, S.; GABLER, S.: Ein neues Stichprobendesign für telefonische Umfragen in Deutschland. In GABLER, S.; HÄDER, S.; HOFFMEYER-ZLOTNIK, J.H.P. (Hrsg.): Telefonstichproben in Deutschland. Wiesbaden [VS Verlag] 1998, S. 69-88.

HÄDER, S.; GLEMSER, A.: Stichprobenziehung für Telefonumfragen in Deutschland. In DIEKMANN, A. (Hrsg.): Methoden der Sozialforschung. Wiesbaden [VS Verlag] 2006, S. 148-171.

HÄDER, M.; HÄDER, S.: Telefonbefragungen über das Mobilfunknetz: Konzept, Design und Umsetzung einer Strategie zur Datenerhebung. Wiesbaden [VS Verlag] 2009. 
HÄDER, S.; HÄDER, M.; GRAESKE, J.; KUNZ, T.; SCHNEIDERAT, G.: Realisierung der Stichprobe. In HÄDER, M.; HÄDER, S. (Hrsg.): Telefonbefragungen über das Mobilfunknetz Konzept, Design und Umsetzung einer Strategie zur Datenerhebung. Wiesbaden [VS Verlag] 2009, S. 71-82.

HÄDER, S.; HÄDER, M.; SCHMICH, P. (Hrsg.): Telefonumfragen in Deutschland. Wiesbaden [Springer VS] 2019.

HÄDER, S.; SAND, M.: Telefonstichproben. In HÄDER, S.; HÄDER, M.; SCHMICH, P. (Hrsg.): Telefonumfragen in Deutschland. Wiesbaden [Springer VS] 2019, S. 113-151.

HÄDER, S.; SCHNEIDERAT, G.: Antwortbereitschaft bei Mobilfunkumfragen. In Sozialwissenschaftlicher Fachinformationsdienst (soFid), Methoden und Instrumente der Sozialwissenschaften, 2, 2009, S. 41-50

HECKEL, C.: Erstellung der ADM-Telefonauswahlgrundlage. In GABLER, S.; HÄDER, S. (Hrsg.): Telefonstichproben: Methodische Innovationen und Anwendungen in Deutschland. Münster [Waxmann] 2002, S. 11-31.

HECKEL, C.: Weiterentwicklung der ADM-CATI-Auswahlgrundlagen. In GABLER, S.; HÄDER, S. (Hrsg.): Mobilfunktelefonie - eine Herausforderung für die Umfrageforschung. Mannheim [GESIS-ZUMA] 2007, S. 25-37.

HEINZ, L.: Festnetzsubstitution durch Mobilfunk: Entwicklung und Tendenzen der soziodemografischen Merkmale bei exklusiven Mobil- und Festnetznutzern. In HÄDER, M.; NEUMANN, R. (Hrsg.): Auswahlprobleme und Antwortverhalten bei Telefonbefragungen (Dresdner Beiträge zur Soziologie, 5). Münster [MV-Wissenschaft] 2015, S. 65-88. HOFFMANN, H.: Kombinierte Stichproben für Telefonumfragen - Ansätze in Europa. In: ZUMA-Nachrichten Spezial, 13, 2007, S. 47-58.

HOOPS, C.; MICHAEL, T.: Mobile and Hard-to-Reach Voters. In: Survey Practice, 8(4). doi:10.29115/SP-2015-0020

HUNSICKER, S.; SCHROTH, Y.: Die Kombination von Mobilfunk- und Festnetzstichproben: Eine praktische Anwendung des Dual-Frame-Ansatzes. Methoden-Daten - Analysen, 1(2), 2007, S. 161-182.

HUNSICKER, S.; SCHROTH, Y.: Dual-Frame-Ansatz in politischen Umfragen (Arbeitspapiere der Forschungsgruppe Wahlen e.V., 2). Mannheim [Forschungsgruppe Wahlen e.V.] 2014. https://www.forschungsgruppe.de/Publikationen/dual_frame_A2_2014.pdf KELLER, F.: Archäologie der Meinungsforschung: Mathematik und die Erzählbarkeit des Politischen. Konstanz [UVK] 2001.

$\mathrm{KOCH}, \mathrm{A}$. : Wenn "mehr" nicht gleichbedeutend mit „besser" ist: Ausschöpfungsquoten und Stichprobenverzerrungen in allgemeinen Bevölkerungsumfragen. In: ZUMA-Nachrichten, 42, 1998, S. 66-93.

KOCH, A.; BLOHM, M.: Nonresponse Bias (GESIS Survey Guidelines). Mannheim [GESIS] 2015. doi:10.15465/gesis-sg_004

KUNZ, T.; FUCHS, M.: Improving RDD Cell Phone Samples. Evaluation of Different Pre-call Validation Methods. Journal of Official Statistics, 28(3), 2012, S. 373-394.

MARHENKE, W.: Telefonanschlußdaten als Auswahlgrundlage. In GABLER, S.; HOFFMEYERZLOTNIK, J.H.P. (Hrsg.): Stichproben in der Umfragepraxis. Opladen [Westdeutscher Verlag] 1997, S. 148-195.

MAURER, M.: Befragtenauswahl bei Telefonumfragen. Wie zuverlässig ist die Geburtstagsmethode? In GEHRAU, V.; FRETWURST, B.; KRAUSE, B.; DASCHMANN, G. (Hrsg.), Auswahlverfahren in der Kommunikationswissenschaft. Köln: [Halem] 2005, S. 203-222. 
MÖHRING, W.; SCHLÜTZ, D.: Die Befragung in der Medien- und Kommunikationswissenschaft: Eine praxistheoretische Einführung (3., vollständig überarbeitete Auflage). Wiesbaden [Springer VS] 2019.

NELLER, K.: Kooperation und Verweigerung: eine Non-Response-Studie. In: ZUMANachrichten, 57, 2005, S. 9-36.

OLIVER, L.: River Sampling Non-Probability Sampling in an Online Environment. 13.11.2011. Center for Information-Based Decision Making and Marketing Research. 2011. http://lexolivier.blogspot.com/2011/11/river-sampling-non-probability-sampling.html

RENDTEL, U.: Gutachten zur Repräsentativität von Online-Umfragen. 15.11.2018. https://civey.com/ueber-civey/unsere-methode/artikel/gutachten-zur-repraesentativitaet-von-online-umfragen

SAND, M.: Dual-Frame-Telefonstichproben: Entwicklung, Handhabung und Gewichtung (GESIS-Technical Reports 2014|02). Mannheim, Köln [GESIS - Leibniz-Institut für Sozialwissenschaften] 2014.

SAND, M.: Dual-Frame-Telefonstichproben: Gewichtung im Falle von Device-Specific Nonresponse. In SCHUPP, J.; WOLF, C. (Hrsg.): Nonresponse Bias: Qualitätssicherung sozialwissenschaftlicher Umfragen. Wiesbaden [Springer] 2015, S. 133-160.

SAND, M.: Evaluierung von HLR-Lookup-Verfahren. Erste Ergebnisse aus dem Projekt VermIn. In EIFLER, S.; FAULBAUM, F. (Hrsg.): Methodische Probleme von Mixed-Mode-Ansätzen in der Umfrageforschung. Wiesbaden [Springer] 2017, S. 211-237.

SAND, M.: Gewichtungsverfahren in Dual-Frame-Telefonerhebungen bei Device-Specific Nonresponse (GESIS-Schriftenreihe, 20). Köln [GESIS] 2018. doi:10.21241/ssoar.60293

SAND, M.; GABLER, S.: Gewichtung von (Dual-Frame -) Telefonstichproben. In HÄDER, S.; HÄDER, M.; SCHMICH, P. (Hrsg.): Telefonumfragen in Deutschland. Wiesbaden [Springer VS] 2019, S. 405-424.

SChaetz, N.; LeIßnNer, L.; PORTen-Ché, P.; EMmer, M.; StRIPPel, C.: Politische Partizipation in Deutschland 2019 (Weizenbaum Report, 1). Berlin: Weizenbaum Institute for the Networked Society. 2020. doi:10.34669/wi.wr/1

SCHNAUBER, A.; DASCHMANN, G.: States oder Traits? Was beeinflusst die Teilnahmebereitschaft an telefonischen Interviews? In: Methoden, Daten, Analysen, 2(2), 2008, S. 97123.

SCHNELL, R.: Nonresponse in Bevölkerungsumfragen: Ausmaß, Entwicklung und Ursachen. Opladen [Leske+Budrich] 1997.

SCHNELL, R.: Warum ausschließlich online durchgeführte Bevölkerungsumfragen nicht repräsentativ sind. 27.11.2018. https://www.marktforschung.de/dossiers/themendossiers/repraesentativitaet-und-zufallsstichprobe/dossier/warum-ausschliesslich-online-durchgefuehrte-bevoelkerungsumfragen-nicht-repraesentativ-sind/

SCHLINZIG, T.; SCHNEIDERAT, G.: Möglichkeiten zur Erhöhung der Teilnahmebereitschaft bei Telefonumfragen über Festnetz und Mobilfunk. In WEICHBOLD, M.; BACHER, J.; WOLF, C. (Hrsg.): Umfrageforschung: Herausforderungen und Grenzen. Wiesbaden [VS Verlag] 2009, S. 21-43.

SCHNEIDERAT, G.; SCHLINZIG, T.: Das Mobilfunktelefonverhalten in der Allgemeinbevölkerung. In HÄDER, M.; HÄDER, S. (Hrsg.): Telefonbefragungen über das Mobilfunknetz: Konzept, Design und Umsetzung einer Strategie zur Datenerhebung. Wiesbaden [VS Verlag] 2009, S. 99-119. 
SCHNEIDERAT, G.; SCHLINZIG, T.: Mobile- and Landline-Onlys in Dual-Frame-Approaches: Effects on Sample Quality. In HÄDER, S.; HÄDER, M.; KÜHNE, M. (Hrsg.): Telephone Surveys in Europe: Research and Practice. Berlin, Heidelberg [Springer] 2012, S. 121-143.

SCHUPP, J.; WOLF, C.: Nonresponse Bias: Qualitätssicherung sozialwissenschaftlicher Umfragen. Wiesbaden [Springer] 2015.

StAdTMÜlleR, S.; SILBER, H.; DAIKELER, J.; MARTIN, S.; SAND, M.; ... ZABAL, A.: Adaptation of the AAPOR Final Disposition Codes for the German Survey Context (GESIS Survey Guidelines). Mannheim [GESIS] 2019. doi:10.15465/gesis-sg_en_026

STÖGBAUER, A.: Ausschöpfungsprobleme telefonischer Umfragen. Eine Zwischenbilanz praktischer gesamtdeutscher Erfahrung. In HÜFKEN, V. (Hrsg.): Methoden in Telefonumfragen. Opladen [Westdeutscher Verlag] 2000, S. 91-104.

STRUMINSKAYA, B.; KACZMIREK, L.; SCHAURER, I.; BANDILLA, W.; GABLER, S.; HÄDER, S.: Identifying Non-Working Numbers in Cell Phone RDD Samples via HRL-Lookup Technology. In: Survey Practice, 4(4), 2011, S. 1-6. doi:10.29115/SP-2011-0021

THIERHOFF, T.: „Repräsentativ“ ist nicht nur ein schmückendes Füllwort. 08.10.2018.

https://www.marktforschung.de/aktuelles/meinung/marktforschung/repraesentativ-istnicht-nur-ein-schmueckendes-fuellwort/

VON DER HEYDE, C.: Das ADM-Telefonstichproben-Modell. In GABLER, S.; HÄDER, S. (Hrsg.): Telefonstichproben Methodische Innovationen und Anwendungen in Deutschland. Münster [Waxmann] 2002, S. 12-45.

VON HERMANNI, H.; LEMCKE, J.: A Review of Reporting Standards in Academic Journals - A Research Note. In: Survey Methods, 2017. https://surveyinsights.org/?p=8625

WILDNER, R.: Herausforderungen bei der Stichprobenziehung in der Markt- und Sozialforschung. 11.11.2020. https://www.horizont.net/planung-analyse/nachrichten/-herausforderungen-bei-der-stichprobenziehung-in-der-markt--und-sozialforschung-186468 